PAPER

\section{A non-contact mechanical solution for implementing synchronized switching techniques for energy harvesting using reed switches}

To cite this article: Ya Shan Shih et al 2016 Smart Mater. Struct. 25125013

View the article online for updates and enhancements.
Related content

- Analog self-powered harvester achieving
$\frac{\text { switching pause control to increase }}{\text { harvested energy }}$
Kanjuro Makihara and Kei Asahina
- Analytical solutions for galloping-based
$\frac{\text { piezoelectric energy harvesters with }}{\text { various interfacing circuits }}$
Liya Zhao and Yaowen Yang
- Enhanced synchronized switch harvesting:
$\frac{\text { a new energy harvesting scheme for }}{\text { efficientenergy extraction }}$
Hui Shen, Jinhao Qiu, Hongli Ji et al.

Recent citations

$$
\begin{aligned}
& \text { A dual resonant rectilinear-to-rotary } \\
& \text { oscillation converter for low frequency } \\
& \text { broadband electromagnetic energy } \\
& \text { harvesting } \\
& \text { Wei Deng and Ya Wang }
\end{aligned}
$$




\title{
A non-contact mechanical solution for implementing synchronized switching techniques for energy harvesting using reed switches
}

\author{
Ya Shan Shih ${ }^{1,2}$, Dejan Vasic ${ }^{1}$ and Wen Jong $\mathbf{W u}^{2}$ \\ ${ }^{1}$ SATIE, ENS Cachan, Cachan, France \\ ${ }^{2}$ Department of Engineering Science and Ocean Engineering, National Taiwan Univeristy, Taipei, Taiwan \\ E-mail: wjwu@ntu.edu.tw and dejan.vasic@satie.ens-cachan.fr
}

Received 27 April 2016, revised 14 October 2016

Accepted for publication 14 October 2016

Published 11 November 2016

\begin{abstract}
In this work we proposed a new mechanical method of implementing the synchronized switching technique for piezoelectric energy harvesting based on reed switches. Serving as a mechanical displacement monitor and the switch itself, it holds the merit of non-contact, persistence, and the low voltage threshold of merely a single PN junction. However, as all mechanical switches inherits chattering, or bouncing, energy loss and damping on the inversion was caused. To side pass the chattering, three types of electro-mechanical hybrid switches were furthermore developed to stabilize the interfered current flow: resistor-capacitor snubbers, inductor-capacitor snubbers, and silicon controlled rectifiers (SCRs). Each of the method has its merit and suitable working conditions. Comparing to conventional electrical switches, the proposed switches, greatly reduced the switch impedance since the mechanical switch part provides a physically open switch, and the electrical switch part merely consist of either a diode and a MOSFET pair, or a single SCR. Subsequently, the power loss due to the circuit was efficiently eliminated.
\end{abstract}

Keywords: energy harvesting, reed switches, piezoelectric, synchronized switching harvesting on inductor, SSHI

(Some figures may appear in colour only in the online journal)

\section{Introduction}

During recent years, energy harvesting has become a popular subject thanks to the lowered power consumption of newly developed integrated circuits. While the power consumption has been lowered to the scale of micro-watts, harvesting from the ambient environments such as vibration, or thermal energy has become feasible. In contradiction to the welldeveloped solar and wind energy, vibrational energy is less limited in environment since it exists namely everywhere. With vibrational harvesting, autonomous sensors could be more parasitic within major structures which requires monitoring, with less consideration to the weather, or temperature. However, the harvested energy from vibration comes with AC signals. To retain and store the energy, it has to be converted in to DC. Thereby, an interfacing circuit is inevitable.

It is also noteworthy that, followed by the lowering of the power consumption and the miniaturized devices, the system also requires a miniaturized power source to retain its petite dimensions. Extending the idea, the minimized size of the power source also reduces the power output of the generating device, aka energy harvester (EH). To provide enough power, it is a duty to maximize the power output of the $\mathrm{EH}$, and the same time the efficiency of the interfacing circuit.

A conventional method is the full bridge rectifier, so as called the standard energy harvesting (SEH). SEH subjects to energy feed backs, and therefore does not provide the maximum power. To improve energy output of SEH, synchronized switch harvesting ( $\mathrm{SSH}$ ) method was proposed as a 


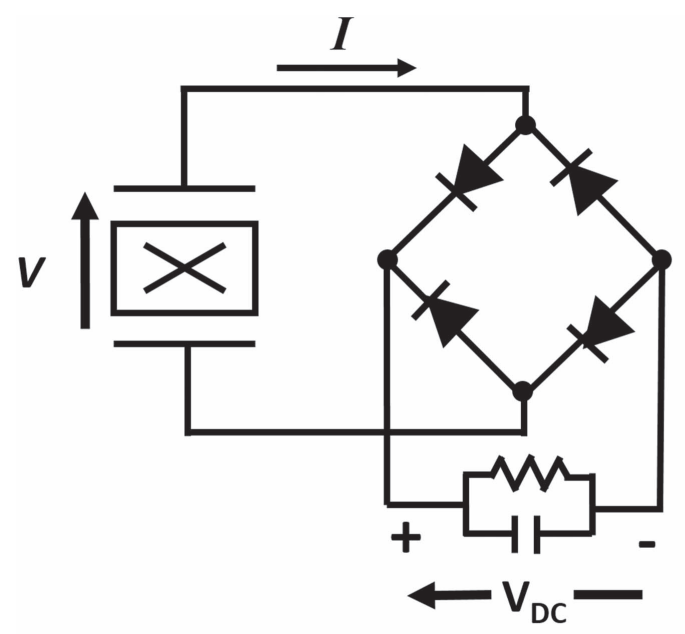

(a)

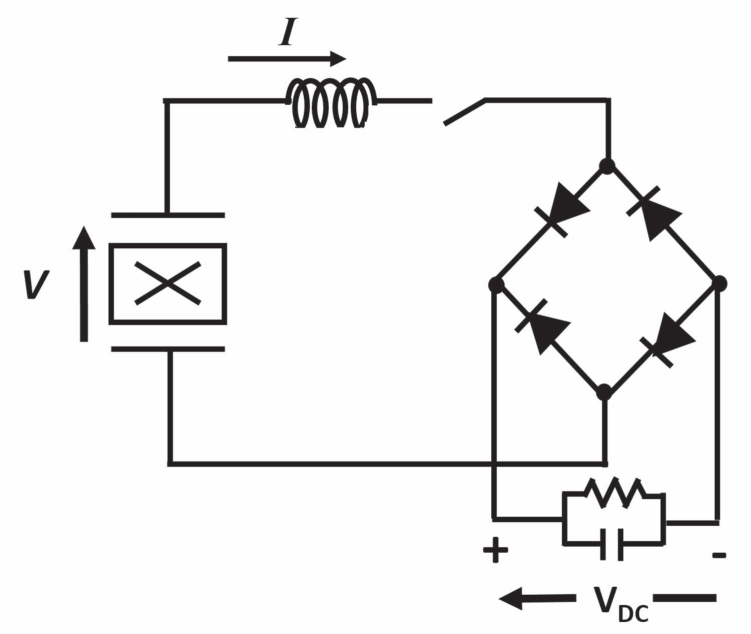

(c)

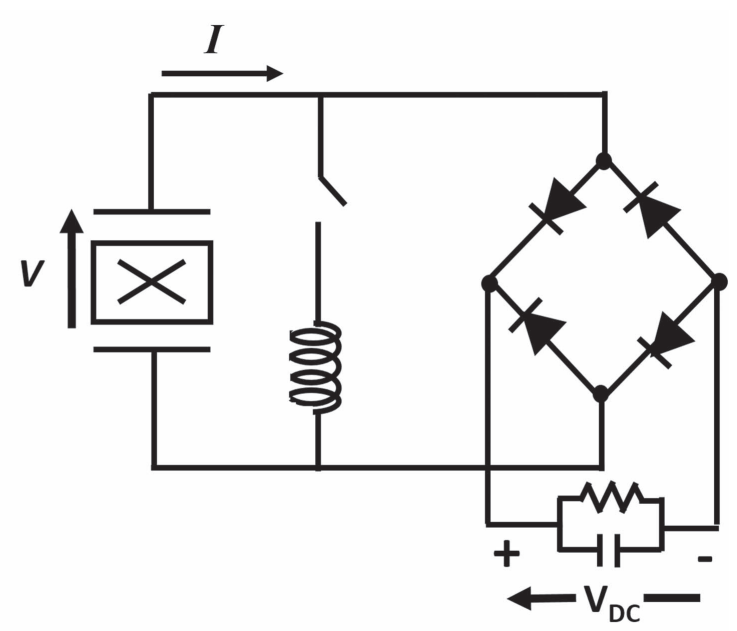

(b)

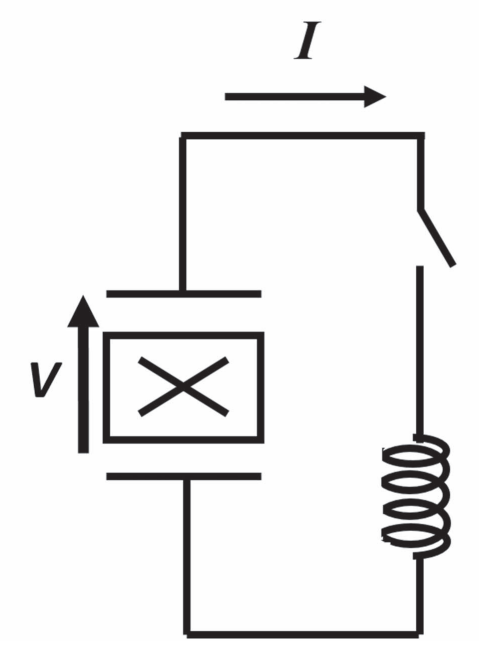

(d)

Figure 1. Circuit topology of (a) SEH (b) P-SSHI (c) S-SSHI (d) LF-P-SSHI.

solution. One of the original structures is the synchronized switch harvesting on inductance (SSHI), which is to launch an LC resonance at the extremes of the displacement. Proposed by Lefeuvre et al during 2004 [1-3] SSHI was intended to be a vibration control method, synchronized switch damping (SSD) [4]. SSHI method was discovered to increase the harvested power by over $400 \%$ in comparison to the SEH circuit on low electro-mechanical coupling systems. By launching the nonlinear processing electronic circuit consisting of a switch and an inductor, a nonlinear effect was conducted on the maximum and the minimum of the mechanical displacement. As the switch turns on, it launches the LC resonance of the inductance and the parasitic capacitance of the piezoelectric patch. This quick resonance was switched off after half the period of the LC resonance, causing an inversion of the piezoelectric voltage. In this way, the voltage and the velocity were forced to be in-phase. By placing the nonlinear components in shunt or series, we can obtain the P-SSHI (parallel SSHI) and the S-SSHI (series SSHI). Other than SSHI, some other techniques of SSH were then proposed as to meet different requirements (figure 1): DSSH [5], SSDCI [6], SECE [7], ESSH [8]... etc [9]. However, a complex circuit also indicates new issues such as power loss due to the various components, threshold voltage, frequency selectivity and also parasitic bandwidth filters. The tradeoffs may pay-off on bulk sized PEHs, however with only the scale of micro-watt output. We aim to design a circuit with simplicity and efficiency. The setup of SSHI is rather simple-to insert a bidirectional switch and inductance in parallel or in series between the piezoelectric patch and the working load, as shown in figure 1. Thus in this work, S-SSHI, P-SSHI and unrectified SSHI, or load free P-SSHI (LF-P-SSHI), are used to prove and investigate the new structure's feasibility.

To control the switch during correct instances, several configurations of smart switches were proposed as the selfpowered electrical solution. It was first proposed by Lallart et al during 2007 [10-12]. The setup consists of a signal buffer composed of a pair of resistance and capacitance. By 
comparing the buffered signal and the piezoelectric output voltage with a transistor, the switch can be conducted using the energy provided by the capacitance within the buffer. This method subjects to the voltage thresholds from the multiple PN junctions within. Shortly after, Liang and Liao then modified the structure to reduce the components as well as the power consumption [13]. With this method, the self-powered synchronized switch was electrically realized with a single patch of piezoelectric material. Later, another approach was proposed by Chen et al [14]. That is, to divide the piezoelectric patch on the beam in to three, one to provide a current sensor, second for an operational comparator, and lastly the harvested patch itself.

Despite the success of the previous works to automatically detect voltage peaks, with the scale of micro-watts, the electrical switches suffer from leakage currents, voltage thresholds of PN junctions, and the power loss due to the complexity of the components. To avoid electrical losses, mechanical switches were proposed. Mechanical methods are threshold-free and can avoid excess component losses. In year 2013, Maiorica et al proposed a zero-threshold rectifying method utilizing the collisions of the beam [15]. With this method, diodes were bypassed and therefore no threshold loss stands. Within the same year, the same team also applied the same method to operate SSH techniques [16]. A similar method was also used by Wu et al to create nonlinear harvesting method in the following year [17]. Other than direct impact, another method was proposed by Liu et al by using the relative location of the electrodes [18] on a bi-stable structure. However, the mechanical conductions which were used in these works attribute to the impacts of the beams or the fractional contact of the electrode. Not to mention the physical limitation of displacement control, the impacting or attrition may cause unwanted damage to the beam itself and accelerate the reduction of the device lifespan.

Therefore another method was proposed by Liu [19] with a parasitic oscillating structure on the tip of the beam. In this method, the main structure would not be damaged during the conducting instances. As far as the main structure is concerned, if the switching element fails, the harvesting can still be working, thence stability can be maintained.

To sidestep the physical impact of the main structure, this work introduces reed switches to serve as the mechanical detector and switch itself. Reed switches are on-shelf, easy to purchase and also can be installed with ease. From the size of bulk to MEMS (micro electronic engineering system), reed switches are controlled by a magnetic field, thus they can be used for non-contact displacement detection. In the energy harvesting application the switch of the SSHI can be controlled by a magnet bonded on the tip of the beam. The launching time of the LC resonance can be tuned via the location of the magnets. It should be however noted, that mechanical switches suffer from the common problem of chattering. The chattering may cause excessive power loss due to the multiple contacts while the reeds are not settled down. To deal with the relay contacts, RC filters and also silicon rectifying control components like thyristors or TRIACs are practical solutions to avoid the noises and

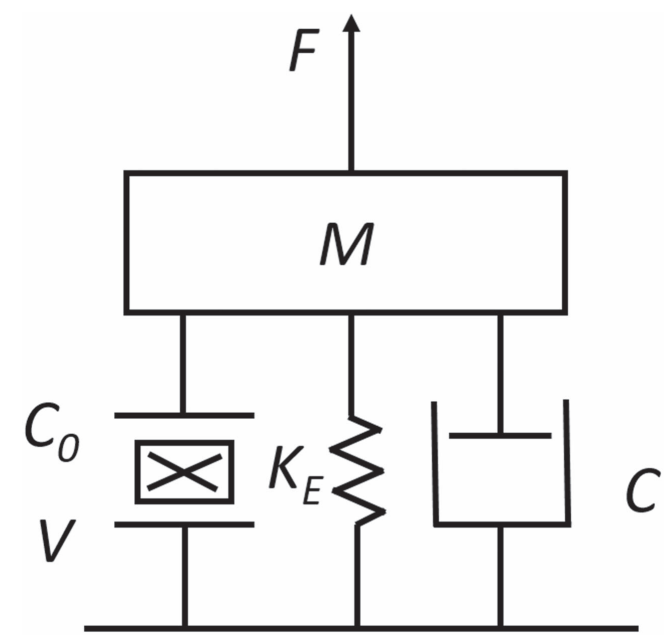

Figure 2. MCK model for a piezoelectric cantilever beam.

excessive signals caused by the phenomenon in power electronics.

In the following sections, we will first introduce the basics of SSHI, and how the reed switch can be applied on SSHI. Problems caused by the reed switch is subsequently pointed out: chattering and phase leading. Three electrical solutions to resolve the chattering are then presented. Experiments performed are then depicted to prove the working mechanism, sort out and understand the power efficiency of each solution. Lastly, the discussion and conclusions are presented.

\section{SSHI for piezoelectric energy harvesting}

In this section, theoretical models of piezoelectric beam system, SEH and SSHI for piezoelectric energy harvesting models are introduced.

Firstly, the mechanism of the piezoelectric beam can be represented in to a mass-spring-damper model as shown in figure 2. The electromechanical coupling equation for a piezoelectric element bonded on the beam structure can be described with equation (1) and (2) [9] where $u$ stands for the beam displacement, $F$ represents the piezoelectric force due to the elasticity and converse piezoelectric action of the piezo patch, $C$ for the equivalent damping factor, $K_{E}$ the short circuited stiffness, and $M$ is the equivalent mass. $I$ stands for the output current, $V$ the piezoelectric output voltage of the piezoelectric element, $C_{0}$ the clamped capacitance and $\alpha$ the force factor of the piezoelectric patch. To further depict the energy relation equation (2) was multiplied by the voltage to obtain equation (4). The integration starts from the time $t_{0}$ to $t_{0}+\tau$ within the period of working frequency. Equation (3) depicts the energy provided to the structure in relation to given excitation force and displacement. The converted energy will be stored into the piezoelectric element in $C_{0}$ and also delivered to the circuit through the circuit, which is expressed as equation (4).

$$
M \ddot{u}(t)=F-\alpha V-K_{E} u-C \dot{u},
$$




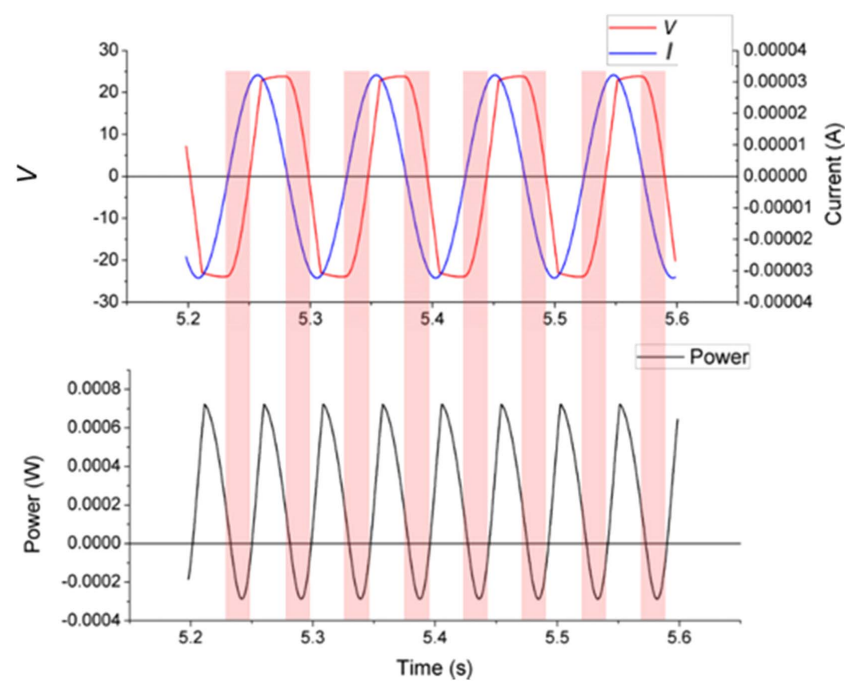

Figure 3. Power feedback as a result of current-voltage phase difference. The red curve $V$ is the piezoelectric voltage. The blue curve indicates the current $I$ flowing through the entire circuit.

$$
\begin{gathered}
I=\alpha \dot{u}-C_{0} \dot{V}, \\
\frac{1}{2} M\left[(\dot{u})^{2}\right]_{t_{0}}^{t_{0}+\tau}+C \int_{t_{0}}^{t_{0}+\tau}(\dot{u})^{2} \mathrm{~d} t+K_{E}\left[u^{2}\right]_{t_{0}}^{t_{0}+\tau} \\
=\int_{t_{0}}^{t_{0}+\tau} F \dot{u} \mathrm{~d} t-\alpha \int_{t_{0}}^{t_{0}+\tau} V \dot{u} \mathrm{~d} t, \\
\alpha \int_{t_{0}}^{t_{0}+\tau} V \dot{u} \mathrm{~d} t=\int_{t_{0}}^{t_{0}+\tau} V I \mathrm{~d} t+\frac{1}{2} C_{0}\left[V^{2}\right]_{t_{0}}^{t_{0}+\tau} .
\end{gathered}
$$

\subsection{SEH circuit}

Figure 3 shows the typical waveforms of SEH. Due to the rectifier, we can see that there is a phase difference between the rectifier loaded output piezoelectric voltage (Vrect) and the piezo current $I$. Consequently, in the output power curve, there are time during the power is negative (colored zone), and thus the average output power is decreased.

The maximal power output of SEH during constant force can be expressed with the equation as follows [5]:

$$
\begin{gathered}
P_{F, \text { standard }}=\frac{R_{L} \alpha^{2}}{\left(R_{L} C_{0} \omega+\pi / 2\right)^{2}} \times \frac{F^{2}}{C+\frac{2 \alpha^{2}}{\left(R_{L} C_{0} \omega+\pi / 2\right)^{2}},} \\
P_{u, \text { standard }}=\frac{R_{L} \alpha^{2}}{\left(R_{L} C_{0} \omega+\pi / 2\right)^{2}} \times \omega^{2} u_{M}{ }^{2},
\end{gathered}
$$

where $R_{L}$ is the optimal load of SEH.

$$
R_{L}=\frac{\pi}{2 \omega C_{0}}
$$

When expressing with respect to the displacement, equation (6) can be used.

\section{2. $\mathrm{SSH}$ on inductor techniques}

To avoid the negative power caused by the phase difference, synchronized switching techniques are applied. By launching a half-cycled LC resonance on the displacement extremes

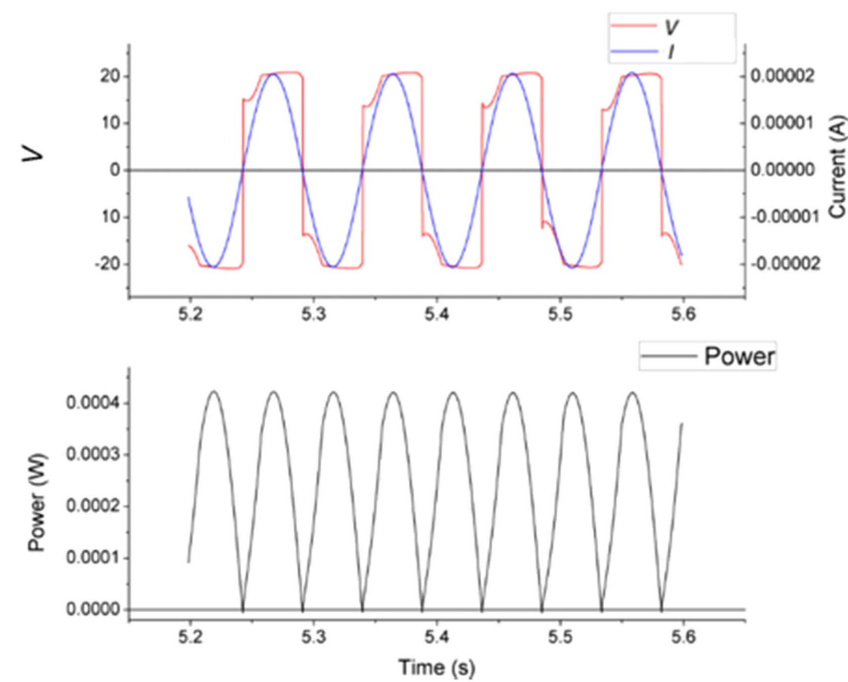

Figure 4. Reduced power feed-back by SSH techniques, by forcing the current and voltage to be in-phase. The red curve $V$ denotes the piezoelectric voltage, and the blue curve $I$ denote the current flowing out of the piezoelectric element.

with a synchronized switch, an inversion of the voltage occurs. The inversion of the piezoelectric voltage therefore greatly reduces the time difference between the voltage and the speed. Resulting in the reduction of negative power which would flow back to the piezoelectric patch (figure 4). On the other hand, the voltage was also increased due to the LC resonance, which also leverages the power output.

For P-SSHI which is shown in figure 1(b), the switchinductor pair is inserted in parallel to the piezoelectric patch and the rectifier. As the voltage comes to its extreme, the current comes to its zero point, and the energy transfer is zero. On this point, the switch is conducted, and a LC resonance is launched. The on switching instance continues for half of the LC resonance period $t_{s}$, which is designed to be much smaller than the source frequency. Nevertheless, we cannot omit the inner resistances of the components which cause damping to the LC resonances. The loss ratio is usually denoted as $\gamma$, where the inversed voltage can be obtained by the product of voltage before inversion and the coefficient. It could be determined with equation (9), where $Q_{i}$ is the quality factor of the resonance.

$$
\begin{gathered}
t_{s}=\pi \sqrt{L C_{0}}, \\
\gamma=e^{-\frac{\pi}{2 Q_{i}}} .
\end{gathered}
$$

Similar to P-SSHI, S-SSHI inserts the inductor-switch pair in series in between the piezoelectric element and the rectifier, the circuit can be found in figure 1(c). The switching instance is identical to that of the P-SSHI, that is, on the displacement extremes. On the displacement extremes, the electrostatic charges are maximal. As the switch is conducted, the current flows through the inductor to the regulating capacitance. One should notice that the experimental $\gamma$ value of the S-SSHI should be obtained with $V_{\mathrm{DC}}$, which is the 


\section{Reed Switch Structure}

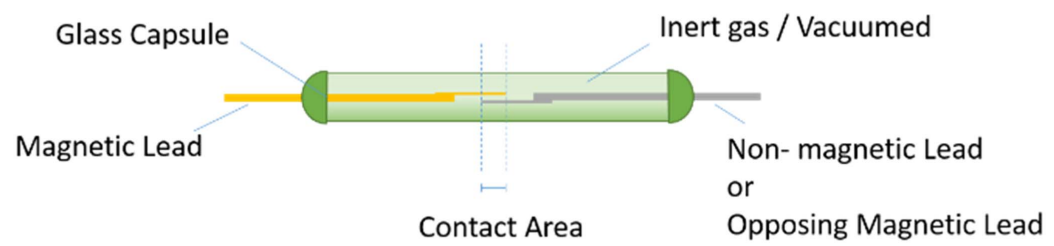

(a)

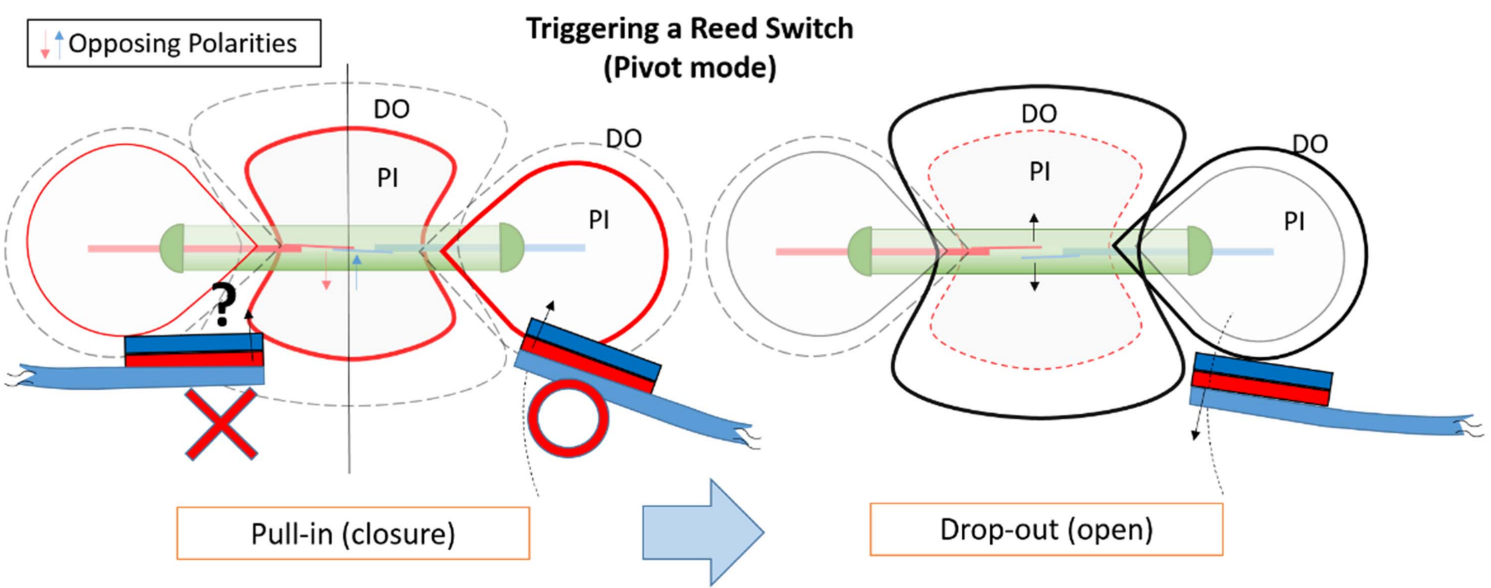

(b)

Figure 5. (a) Reed switch structure (b) triggering and releasing a reed switch with a magnet. On the left most shows a possible occasion of triggering two PI zones at once.

rectified voltage across the load and capacitor of the rectifier, as reference, as shown in figure 1(c).

\section{Reed switch based SSHI for piezoelectric energy harvesting}

In this section, we are going to introduce the operating concepts of using reed switch on SSHI. Also, this section mentions about the chattering and the phase leading problems which were inevitable in reed switch applications.

The reed switch we applied in this work is one of the most commonly seen among the various types: the glass sealed reed switch. A glass sealed reed switch is composed of two ferromagnetic blades, sealed within vacuumed glass capsules or inert gas-filled capsules. When suitable magnetic field approaches, either by magnets or a current flowing coil, the overlapping area of the two reed blades would come to closure, resulting in the conduction. To contact the reed blades with a singular pole, each of the blades should respond in opposite directions. Thus, the two blades are differentiated in to different leads, one positively poled magnetic lead, and the other lead non-magnetic or negatively polarized. Figure 5(b) shows a schematic diagram of how the magnet alternates the switch: As the magnetic field approaches the pull in (PI) zone, the two reeds are polarized inversely, and therefore attracted to each other for contact.
Due to magnetic hysteresis, the magnet has to leave the drop out (DO) zone to release the contacting blades. There are also side lobes on both sides of the switch with identical functions. Since there are overlapping areas of the main lobe and the side lobes on the moving trace of beam displacement, it is occasional that the magnet may trigger either the side lobe first or the main lobe first. In this case the switching becomes unstable. Thus, in this work we choose to operate the reed switch by only using the side lobes to avoid multiple triggering.

\subsection{Implementing reed switch on SSHI}

We are then able to alternate the states of the switch taking the advantage of the aforementioned characteristics, using the side lobes to trigger the switch. As simple as such: place magnets on the tip of the cantilever beam, and a pair of reed switches below and above. As the beam vibrates to its extremes, the movement of the tip magnets would turn on the switch as approaching the PI zone and off as it leaves. A DC input testing experiment using a power supply can be found in figure 6(a). It is to observe the duty and the switching respond to the vibration. The DC voltage of $V_{H}$ is provided by the power supply, when the switch conducts, the output voltage $V_{\mathrm{O}}$ would change from 0 voltage to $V_{H}$. The distance between the beam tip at rest and the PI zone is denoted as $d$, and the radius of the PI zone $r_{\mathrm{PI}}$, whilst the distance between the beam and reed is 


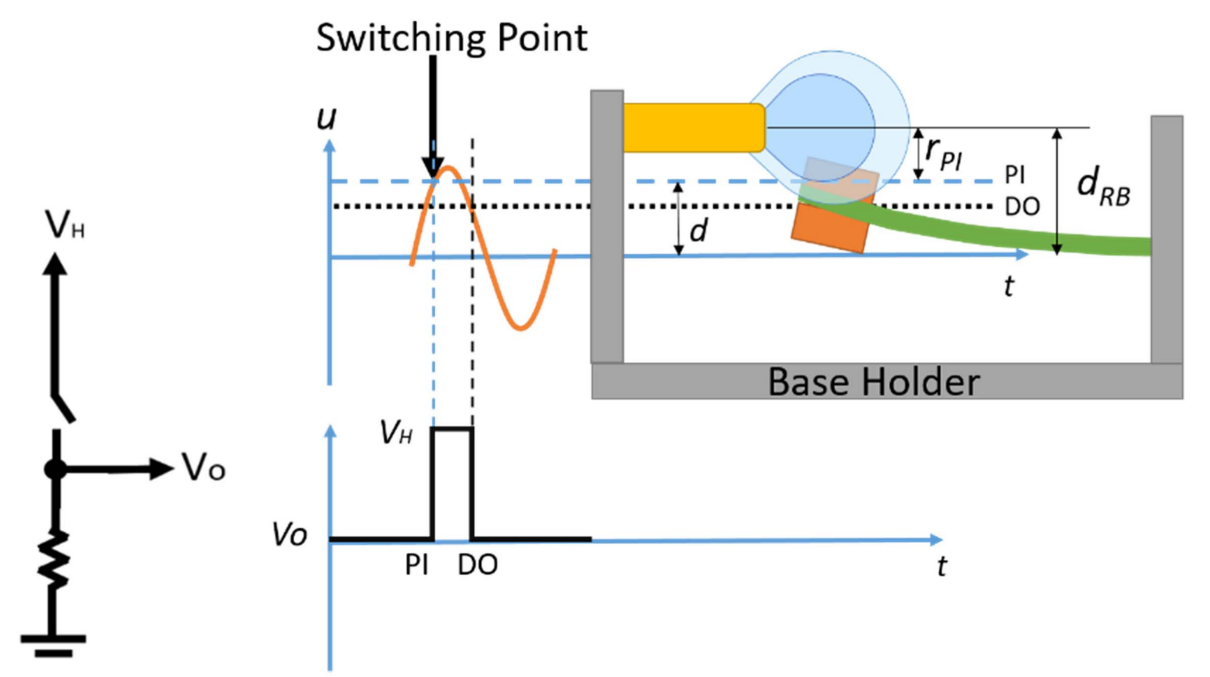

(a)

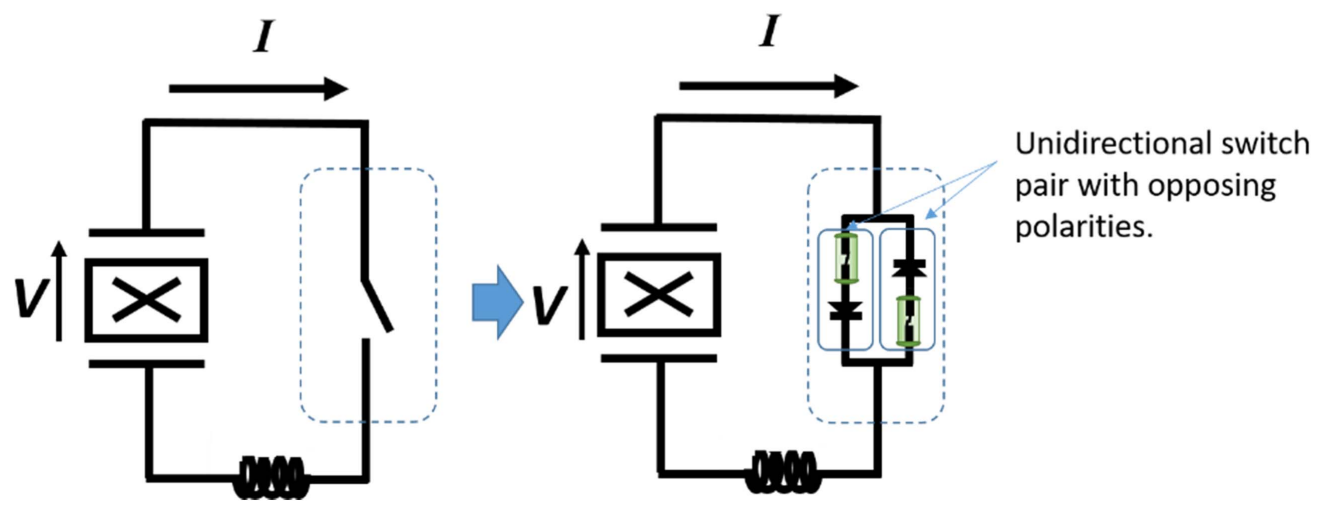

(b)

Figure 6. (a) Operating concept of the displacement monitoring reed switch, $u$ denotes the beam tip deflection, $d$ the distance between the PI zone and the beam, $d_{\mathrm{RB}}$ the reed-beam distance (around $20 \mathrm{~mm}$ ) and $r_{\mathrm{PI}}$ denotes the radius of the PI zone, in the scales of approximately $5 \mathrm{~mm} . V_{H}$ is the DC voltage input using a power supply, and $V_{\mathrm{O}}$ is the output voltage in this experiment (b) LF-P-SSHI using a bi-directional switch consisting unidirectional reed-diode pair with opposing polarities.

denoted as $d_{\mathrm{RB}}$. From the experiment, we find the on duty of the output voltage is determined by the distance between the PI and DO zone, and also the tip deflection of the beam $u$. One should note that, to invert the voltage, the switch should only conduct for half an LC resonance period. To achieve this, a diode was used to halt the reversing current, so that the LC resonance would not persist. Thence, each reed-diode pair is a unidirectional switch, and would need to be paired up in opposing directions to form a fully functioning switch. A schematic diagram of the how the switch is substituted by the reed-diode pairs when applied in a load-free short circuited SSHI is shown in figure 6(b), which is a LF-P-SSHI.

Before dipping into the experiment, there are some important parameters that should be identified: the PI, the DO, hysteresis, and the dynamic contact resistance (DCR). When using a permanent magnet, PI is usually measured in distance, millimeters or inch. It could be also measured in field strength miliTesla or Gauss. When using coils, PIs are measured by, currents through the coil, volts across the coil, or ampere turns (ATs). The DO could be measured in similar means as the PI measurement. It is worth noting that, the PI range and the DO range exists some difference due to the hysteresis. The hysteresis is usually expressed by DO/PI in percentage. Due to the difference of the reed switch design and fabrication, the hysteresis varies. The three parameters are to be used to decide the distance between the tip deflection and the reed switch itself. The ideal switching position should be on the extremes of the displacement. Otherwise phase differences or no switching would occur. Nevertheless, it is impossible to ensure that all displacements are perfectly switching on the extremes during actual usages. Thereby, a margin zone of the relationship between the tip deflection and the switch location should be defined. 


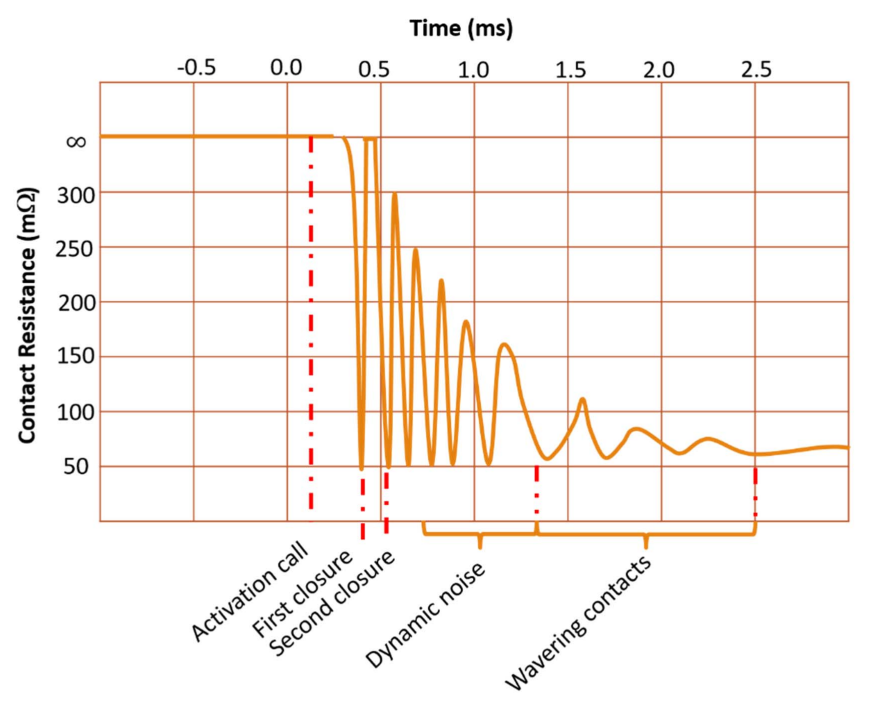

(a)

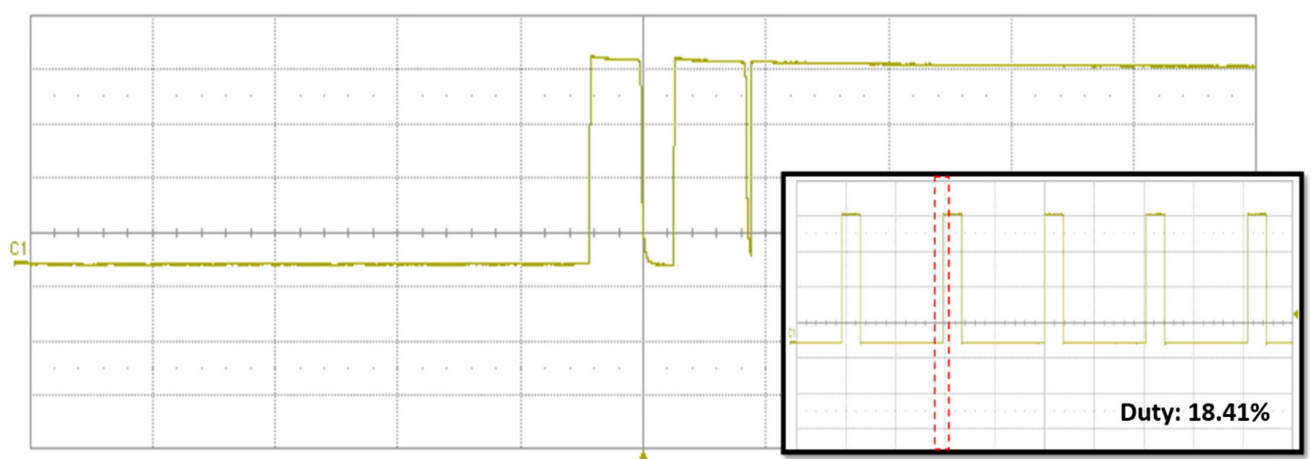

(b) Negative time lag

Figure 7. (a) Dynamic contact resistance in respond to the contact bounce, redrawn from electronics [20]. (b) The screenshot of the oscilloscope waveform for the chattering. The vertical axis is in $1 \mathrm{~V} /$ div and the time scale is $50 \mu \mathrm{s} /$ div.

\subsection{The chattering problem}

As mentioned in the previous section, mechanical switches often suffer from chattering. To investigate, the chattering can be represented with the DCR. Figure 7(a) shows a typical DCR during a call-close period. After the activation call, there is a first closure, and a reopening, then to the second closure, and the resistance wavers until settle. The wavering noise is cause by the material bounce, but not opening. Typically, the bouncing occurs for around $100 \mu \mathrm{s}$, while the following noise settles within $0.5 \mathrm{~ms}$.

Using the example experiment we have introduced in figure 6(a), we obtain figure $7(\mathrm{~b})$ as the result. The high output indicates the closure of the switch, which has the duty cycle of $18.41 \%$. Zooming into the time axis, results show the chattering: there are two openings which are in subsequent to the chattering.

\subsection{Phase difference effects on SSH techniques}

As mentioned by Lallart et al [21] that, in practical applications, the switching instance is usually not optimal. For example, as shown in the left of figure 8(a), smart switches use a buffering capacitance with a small phase lag to trace the piezoelectric voltage. A transistor was used to compare the difference between the two signals of buffer voltage $V_{\text {Buf }}$ and piezoelectric voltage $V$. Subsequently, a threshold voltage $V_{\mathrm{TH}}$ is required to trigger the comparator with one or more PN junctions, depending on the switch design. The threshold voltage then causes the real switching point to be delayed, as depicted in the right of figure 8(a). Applying the reed switch also encounters time difference. In contrast to the smart switch lag, the reed switch causes a leading difference due to its mechanical position. As depicted in the figure $8(\mathrm{~b})$, in the good zone, the beam deflection is large enough to reach the PI zone, so as to conduct the switch. However, there is a range of over shooting. With deflection too much would cause large phase leading conduct. The phase leading conduct would cause the deduction of power harvested. The relationship between the phase lead and deflection can be found in equation (15), whist the power relation can be found in equations (16)-(18). When deflection is too low the switch cannot be conducted. Therefore, too large and too small deflections are inadequate positions. The time difference $\tau$ can also be expressed with phase difference $\theta$. On the other 

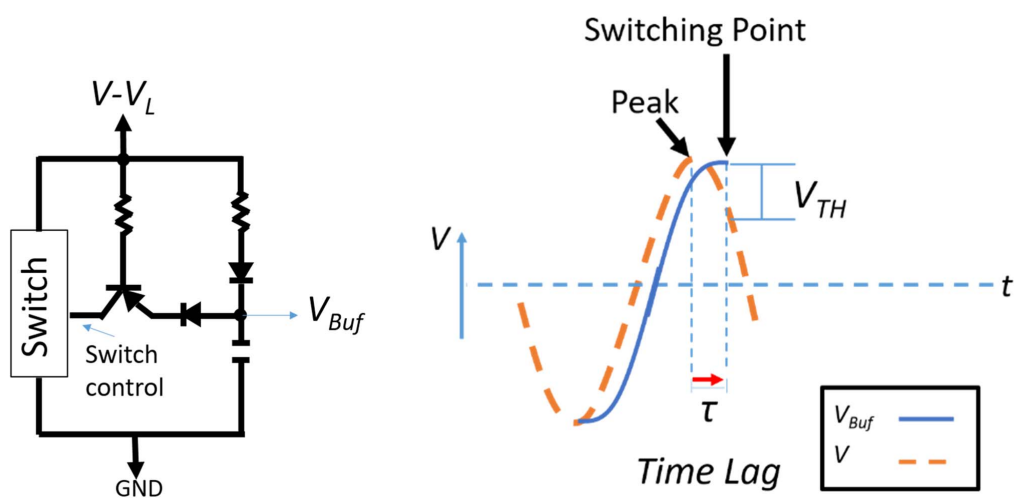

(a)

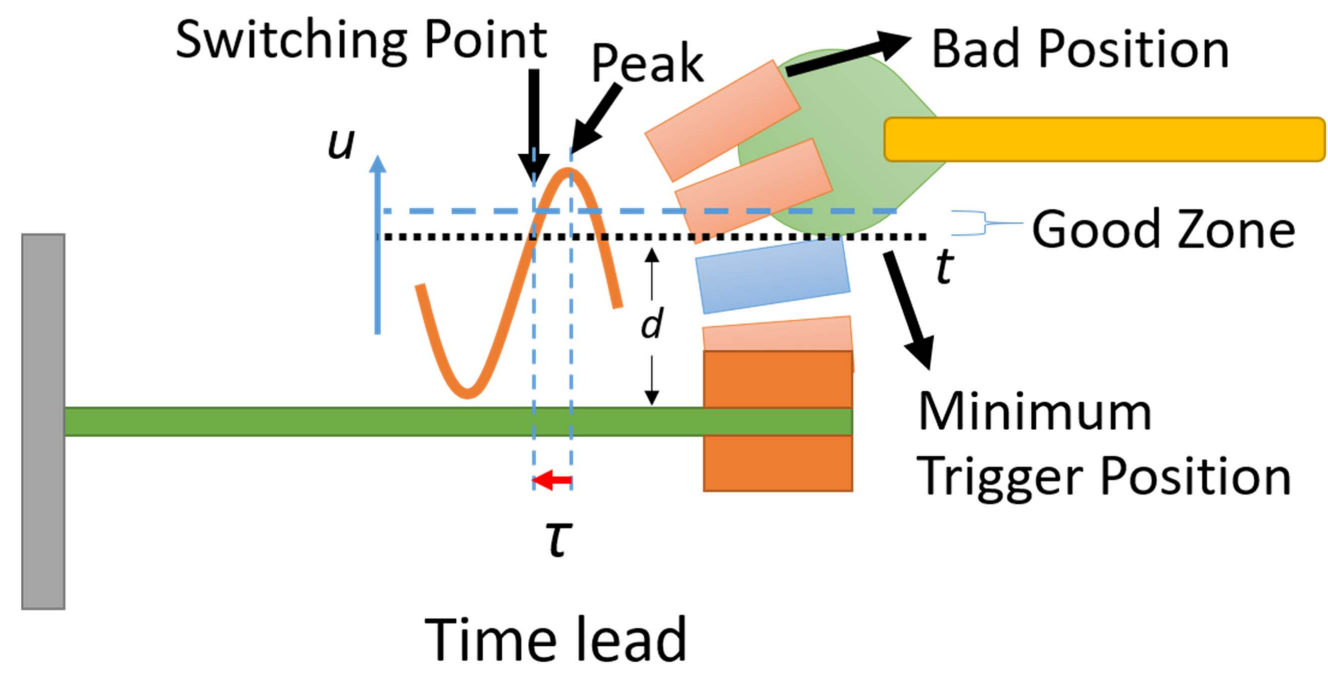

(b)

Figure 8. (a) Time lag effect due to voltage thresholds of the smart switch (left). The dashed line in the color orange represents the capacitance piezoelectric voltage. The blue solid line shows the buffered voltage. (b) With deflection too much would cause large phase leading conduct. The phase leading conduct would cause the deduction of power harvested. Relationship can be found in equation (15). When deflection is too low the switch cannot be conducted. Therefore, too large and too small deflections are inadequate positions. In the good zone, the beam deflection is large enough to reach the PI zone, so as to conduct the switch. However, there is a range of over shooting.

hand, oscillating with displacement lower than required would not conduct the switch. Thus, there exist a feasible working zone where acceptable phase lead occurs.

For P-SSHI and S-SSHI, the harvested power expressions with optimal switching instance and the phase difference are respectively listed below:

$$
\begin{gathered}
P_{\text {para,opt }}=\frac{4 R_{L} \omega_{r}^{2} \alpha^{2}}{\left[\pi+R_{L} \omega_{R} C_{0}(1-\gamma)\right]^{2}} u_{M}{ }^{2}, \\
P_{\text {para,lag }}=\frac{R_{L} \omega_{r}^{2} \alpha^{2}[1+\cos \theta-\gamma(1-\cos \theta)]^{2}}{\left[\pi+R_{L} \omega_{R} C_{0}(1-\gamma)\right]^{2}} u_{M}{ }^{2}, \\
P_{\text {para,lead }}=\frac{4 R_{L} \omega_{r}^{2} \alpha^{2}}{\left[\pi+R_{L} \omega_{R} C_{0}(1-\gamma)\right]^{2}}\left(u_{M} \cos \theta\right)^{2}, \\
P_{\text {series,opt }}=\frac{4 R_{L} \omega_{r}^{2} \alpha^{2}(1+\gamma)^{2}}{\left[\pi(1-\gamma)+2 R_{L} \omega_{R} C_{0}(1+\gamma)\right]^{2}} u_{M}{ }^{2}, \\
P_{\text {series,diff }}=\frac{4 R_{L} \omega_{r}^{2} \alpha^{2}(1+\gamma)^{2}}{\left[\pi(1-\gamma)+2 R_{L} \omega_{R} C_{0}(1+\gamma)\right]^{2}}\left(u_{M} \cos \theta\right)^{2} .
\end{gathered}
$$

The relationship between the given force, the beam, and the displacement can be found in the original paper [21]. According to the paper, it is noteworthy that for P-SSHI, the phase leading and lag can be expressed as equations (11) and (12). However, for S-SSHI, the phase difference causes symmetric effects for both phase lead and lag, as shown in equation (14). In the above equations, $\omega_{r}$ stands for the resonance frequency. In this application of reed switch, $\cos \theta$ can be identified as:

$$
\cos \theta=d / u_{M}
$$

where $u_{M}$ is the maxima deflection amplitude of the beam. 


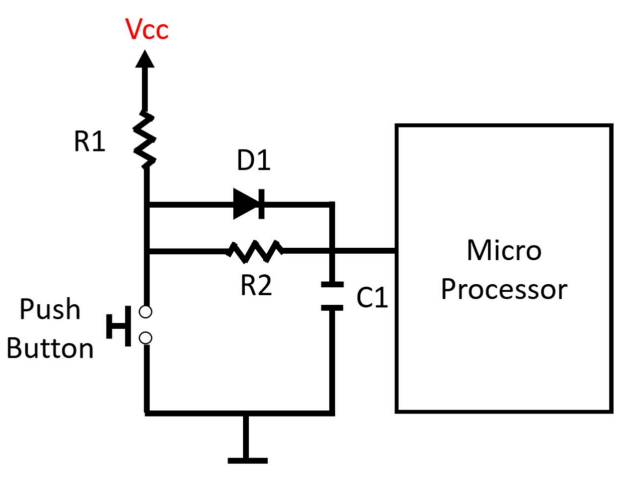

GND

(a)

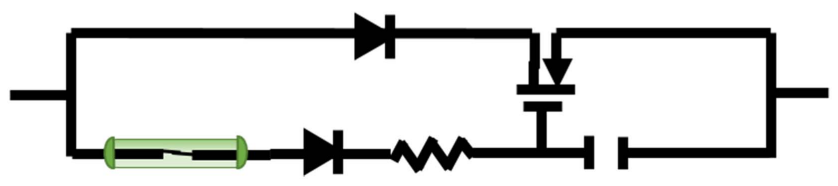

(b)

Figure 9. (a) A commonly seen RC snubber for MCU applications. (b) Adopted unidirectional hybrid configuration of the reed RC snubbed switch.

\section{De-chattering: electro-mechanical hybrid solutions}

In this section we will introduce the electro-mechanical solutions to resolve chattering: the RC and LC snubbers, also the silicon controlled rectifier (SCR). Snubbers are devices used to suppress, or snub, rapid transient phenomena in mechanical or electrical systems such as voltage, pressure or forces. $\mathrm{RC}$ snubber is one of the lowest power consuming and the simplest structure, thus the first choice chosen in our application. Electrical snubbers like RC snubbers can be found in different occasions such as power electronics and also micro-processor mechanism de-bouncing. Other approaches for de-bouncing such as Schmidt Trigger and using logic latches requires operational amplifiers require larger amount of energy.

\subsection{Theoretical development and simulations}

4.1.1. $R C$ snubbers. RC snubbers, are firstly proposed. The adopted hybrid RC snubber switch for de-bouncing is as shown in figure 9(b). It is a unidirectional switch, and therefore a pairing of opposing direction is required when used in application as mentioned. The RC snubbing switch would be denoted as RDR (reed de-bounced by RC) in the following of the text.

We can explain the snubbing mechanism using figure 9(a), which shows a low-level triggered application of microprocessors. The input of the micro processing unit (MCU) is normally high, with the charging of the capacitance from the diode. When the push button is switched on, the voltage level is instantly grounded, and the diode passage is

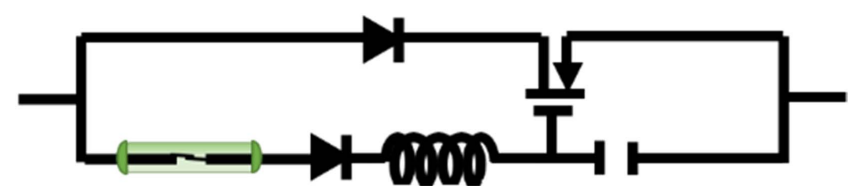

Figure 10. Adopted unidirectional hybrid configuration of the reed LC snubbed switch.

blocked. And the capacitance would discharge through the resistance. Even with contact bouncing, the RC discharge would persist a stable voltage signal, or we can say the signal is snubbed (hereby filtered). In other applications, diodes can be omitted, which will result in the charging and discharging when conducting and detaching, which would slow down the dynamics of re-charging when the switch is off.

The RC snubber is a first order filter and the cut-off frequency can be calculated by: $f=1 /(2 \pi R C)$. In SSHI application (figure 6(b)) RC snubber should be designed to filter the ringing frequency and its cut-off frequency should be after the piezoelectric voltage inversion frequency that is the frequency of the LC circuit: $f=1 /(2 \pi \sqrt{L C})$. The ringing frequency can be observed with experiments. Usually, we find the frequency two or more orders higher than the targeting LC resonance. Thus it is easy to determine the RC values. We should note that the capacitance should be small enough to have high enough corner frequency for the RC snubber. The capacitance value should also be large enough to store enough energy to overcome the gate leakage for the MOSFET to hold enough voltage to turn on the MOSFET. One could use a semi-empirical method of estimating the MOSFET on-power from the component datasheet. The leakage current for the MOSFET in the operating voltage can be obtained from the datasheet and the appropriate range of the capacitance value can be derived accordingly. For instance, in our example in the range of $0.68-68 \mathrm{nF}$.

4.1.2. LC snubbers. The second snubber proposed is the LC snubber. By substituting the resistor to an inductor, the RC first order filter would become a second order filter, the LC filter (figure 10). Since the inductor is ideally non-power consuming, it was expected that the loss would be decreased. Also the spiking signals should be better attenuated due to the characteristic of second order filtering. Thus we expect the LC snubber to be more efficient in comparison to RC snubbers. LC snubbed switch would be denoted as RDI, (reed debounced with inductor-capacitance) in this text.

4.1.3. De-bouncing with SCRs. The last configuration is the SCR (figure 11). SCRs are semi-conductor devices formed with a stack of four layered-PN junctions, usually used to handle large amount of power. It has an appealing characteristic-current latching. That is, the triggering gate current $I_{\mathrm{GT}}$ can only launch the conduction, but cannot determine when to switch off until the device meets its off 


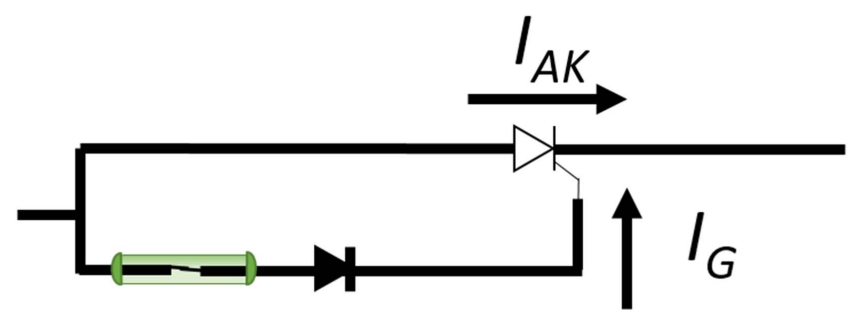

Figure 11. The adopted unidirectional SCR switch.

turning conditions to off-switch the component, there are three methods:

(1) Reverse the voltage over the anode and cathode $V_{\mathrm{AK}}$,
(2) Lower, cut off, or reverse the passing current $I_{\mathrm{AK}}$.

(3) Provide a large amount of reversing gate current $I_{\mathrm{GT}}$ (Gate turn off). This is usually not considered due to the great amount of current.

The latching characteristic meets just what SSH techniques require, and so does the switch-off characteristic-in SSH we wish to stop conduction when the current is lowered or reversed, which naturally happens in SCRs. Despite its latching characteristics, SCRs, as we have mentioned are usually used for power controls, indicating that it may not be suitable for low power applications due to the power loss and high leakage current. Thus, the applied component should be

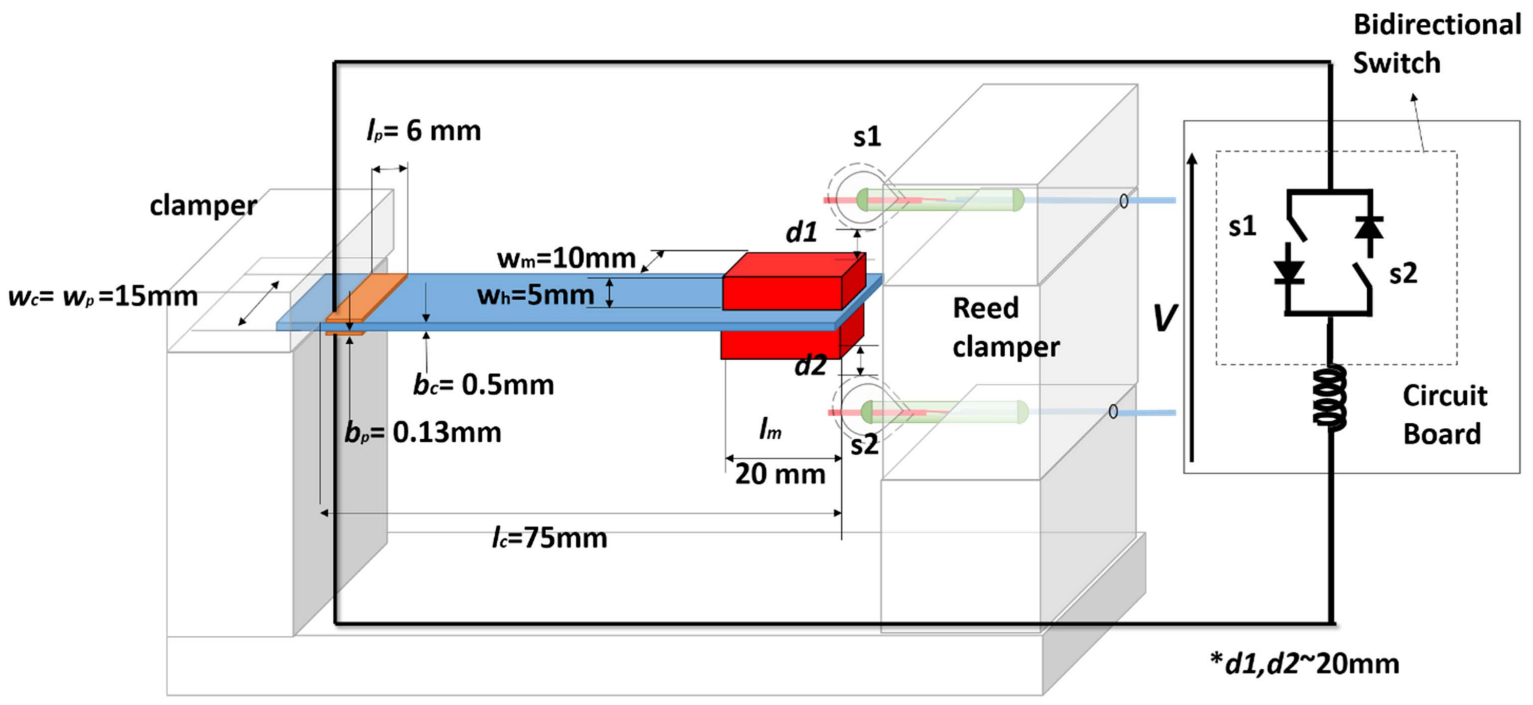

(a)

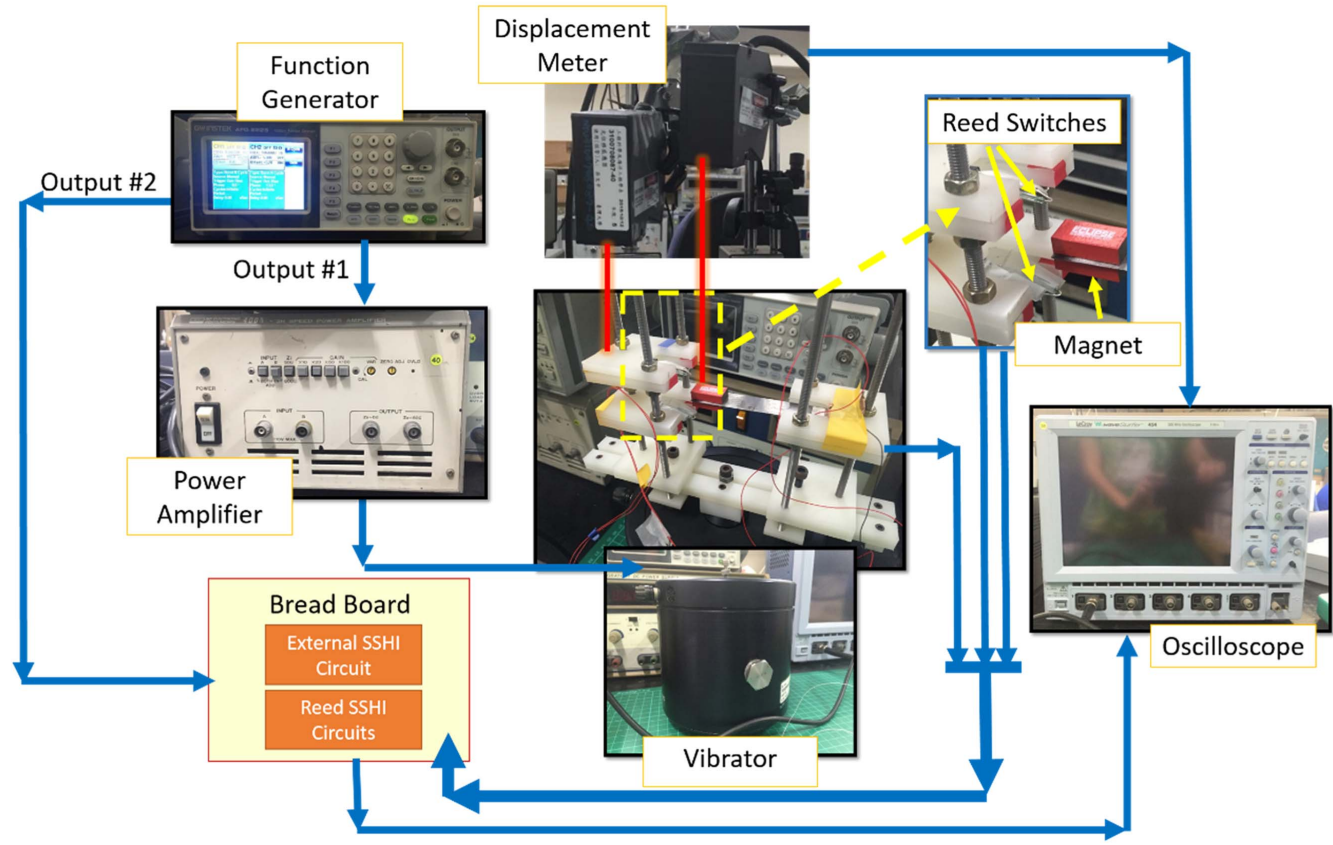

(b)

Figure 12. Experimental setup. (a) Schematic diagram, $d 1$ and $d 2$ are distances of maximum beam deflection to the brink of reed PI zone, which is around $20 \mathrm{~mm}$ (lateral distance also determined by the PI size). (b) Actual setup and connections. 
Table 1. Beam parameters.

\begin{tabular}{|c|c|c|}
\hline & Description & Units \\
\hline Beam dimension & Spring steel & \\
\hline$l$ (length) & 75 & $\mathrm{~mm}$ \\
\hline$w$ (width) & 15 & $\mathrm{~mm}$ \\
\hline$b$ (thickness) & 0.5 & $\mathrm{~mm}$ \\
\hline $\begin{array}{l}\text { Proof } \\
\qquad \text { Mass }\left(l^{*} w^{*} b\right)\end{array}$ & $20^{*} 10^{*} 5$ & Mm (per piece) \\
\hline Proof Mass (mass) & $12 \times 10^{-3}$ & $\mathrm{~kg}$ \\
\hline $\begin{array}{l}\text { Piezoelectric patch } \\
\text { dimension }\end{array}$ & PZT-KA2 & $\begin{array}{l}\text { Eleceram Technology } \\
\text { Co., Ltd } \\
\text { (Manufacturer) }\end{array}$ \\
\hline$l$ (length) & 6 & $\mathrm{~mm}$ \\
\hline$w$ (width) & 15 & $\mathrm{~mm}$ \\
\hline$b$ (thickness) & 0.13 & $\mathrm{~mm}$ \\
\hline Lump parameters & $\begin{array}{l}\text { (Beam } 1+ \\
\text { proof mass) }\end{array}$ & \\
\hline$f_{0}$ & 10.19 & $\mathrm{~Hz}$ \\
\hline$f_{1}$ & 10.21 & $\mathrm{~Hz}$ \\
\hline$C_{O}$ & $0.9 \times 10^{-9}$ & $\mathrm{~F}$ \\
\hline$M$ & $25 e-3$ & $\mathrm{Kg}$ \\
\hline$K$ & 107 & $\mathrm{~N} \mathrm{~m}^{-1}$ \\
\hline$C$ & $2.6 \times 10^{-2}$ & $\mathrm{~N} \mathrm{~s} / \mathrm{m}$ \\
\hline$K_{D}$ & 255.553 & \\
\hline$K_{E}$ & $254.5 \mathrm{e}$ & \\
\hline$Q_{\mathrm{M}}$ & 37.66 & \\
\hline$k^{2}$ & 0.0103 & \\
\hline Lump parameters & $\begin{array}{l}\text { (Beam } 2+ \\
\text { proof mass) }\end{array}$ & \\
\hline$f_{0}$ & 10.496 & $\mathrm{~Hz}$ \\
\hline$f_{1}$ & 10.512 & $\mathrm{~Hz}$ \\
\hline$C_{O}$ & $0.86 \times 10^{-9}$ & $\mathrm{~F}$ \\
\hline$M$ & $26 \times 10^{-3}$ & $\mathrm{Kg}$ \\
\hline$K$ & 80.644 & $\mathrm{~N} \mathrm{~m}^{-1}$ \\
\hline$C$ & $6.183 \times 10^{-2}$ & $\mathrm{~N} \mathrm{~s} \mathrm{~m}^{-1}$ \\
\hline$K D$ & 328.75 & \\
\hline$K E$ & 327.75 & \\
\hline$Q_{\mathrm{M}}$ & 66.42 & \\
\hline$k^{2}$ & 0.0342 & \\
\hline
\end{tabular}

carefully chosen with high sensitivity and low power dissipation. In this experiment, P130AA from STMicroelectronics were chosen for its low triggering gate current $I_{\mathrm{GT}}$, which is merely $0.1 \mu \mathrm{A}$. With a low $I_{\mathrm{GT}}$, it is possible for us to apply the component since that the piezoelectric materials are high impedance energy sources, with high voltage output but limited current.

One should note that for sensitive SCRs like P0130AA, they hold a low reverse gate voltage $V_{\mathrm{RG}}$ of only 8 volts. When the gate-cathode cross voltage exceeds this value, the PN junction breaks down, which conducts the PN junction. Therefore, if excessive voltage is to be supplied, a protecting component should be considered to resolve this incident.
Table 2. Component list used in the experiment.

\begin{tabular}{lll}
\hline Component & Part no., manufacturer & $\begin{array}{l}\text { Component } \\
\text { value }\end{array}$ \\
\hline $\begin{array}{l}\text { Inductor } \\
\begin{array}{l}\text { Snubber } \\
\text { capacitance }\end{array}\end{array}$ & RL181S-104J-RC, Bourns & $\begin{array}{l}100 \mathrm{mH} \\
68 \mathrm{nF}\end{array}$ \\
$\begin{array}{l}\text { Resistance } \\
\text { NMOS: }\end{array}$ & N/A & $10 \sim 100 \mathrm{k}$ \\
& 2N7002, Diode & See datasheet \\
PMOS: & Incorporated & SDS0610, Fairchild \\
SCR & P0130aa, & See datasheet \\
& $\quad$ STMicroelectronics & \\
Diode & BAT 54 W, Infineon & See datasheet \\
Reed switch & RI-27A, Coto technology & See datasheet \\
\hline
\end{tabular}

\section{Experiment}

\subsection{Setup}

As plotted in figure 12(a), a spring steel based cantilever beam was used, epoxy bonded with two piezoelectric patches of PZT-KA2 manufactured by Eleceram Technology Co., Ltd, one on top and the other on the bottom root of the beam. Two magnets were used as the proof mass, located on the tip of the beam. The dimensions and parameters can be found in table 1 . Two beams, beam 1 and beam 2 were used in this experiment, they consist of identical beam dimensions, but different batch of KA-2 piezoelectric patches. The lump parameters were also included in table 1 . The piezoelectric patches are then connected in series, so as to provide higher voltage source. The electrodes of the patches are connected to the SSHI circuit, where, in figure 12(a), a reed diode pair was used as an example of the bidirectional switch. The above mentioned reed switches and the cantilever beam were fixed on a clamper system with tunable $x-y$ rails, as shown in the overall system schematic of figure 12(b).

To compare the output power, externally powered SSHI was used as the reference, which stands for a loss-free switch, despite the fact that it is not. Small losses still exist in the MOSFET switch. However, when driven with voltage high enough, this loss can be neglected. The externally powered SSHI has its driving signal provided by the second output port of the function generator, where the first output is directed to the power amplifier to drive the vibrator. The vibrator will then provide mechanical force for power harvesting. The designed bidirectional switches types were compared, in LFP-SSHI, P-SSHI, and also S-SSHI topologies. The electronic components used in our experiments are listed in table 2.

For measurement, the output signals are then connected to an oscillator, where we could observe the results. The displacements were measured by laser displacement meters, one monitoring the base and the other the tip to measure the tip displacement of the beam and the vibrating acceleration. The beam deflection can be derived by the difference of tip displacement and beam displacement. Current was measured by Tektronix CT-2 probes when applying LF-P-SSHIs. 


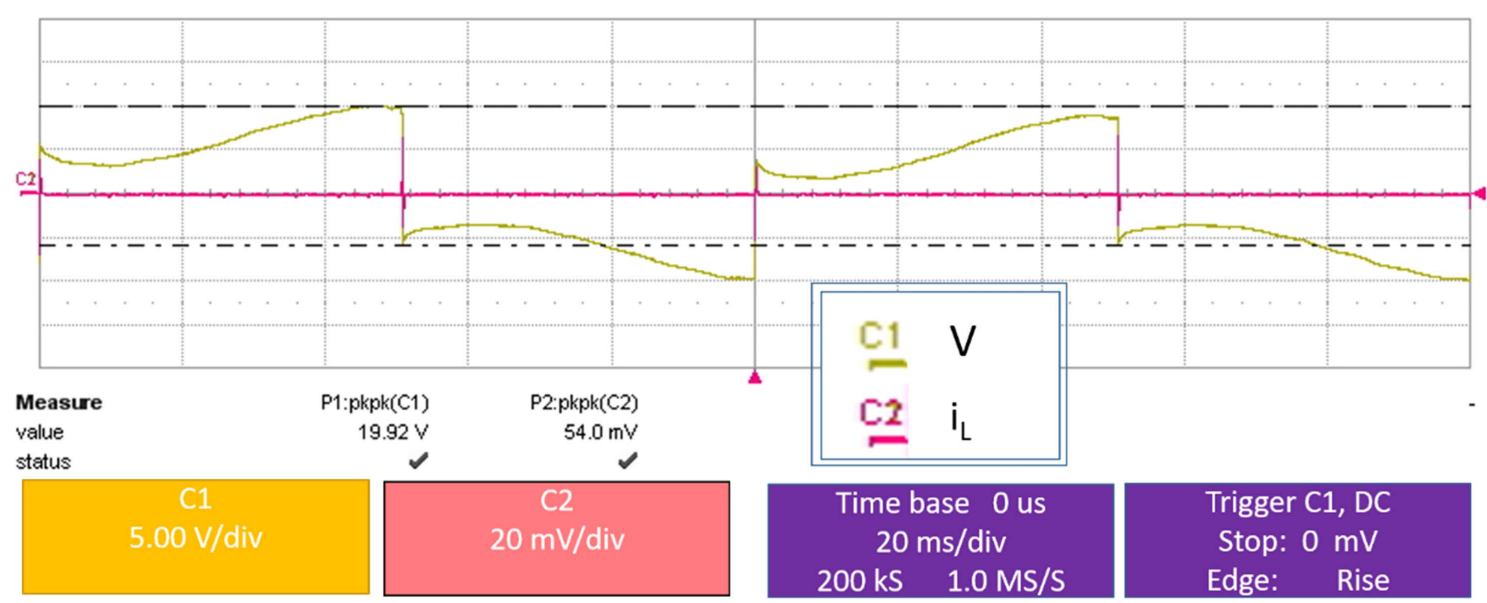

(a)

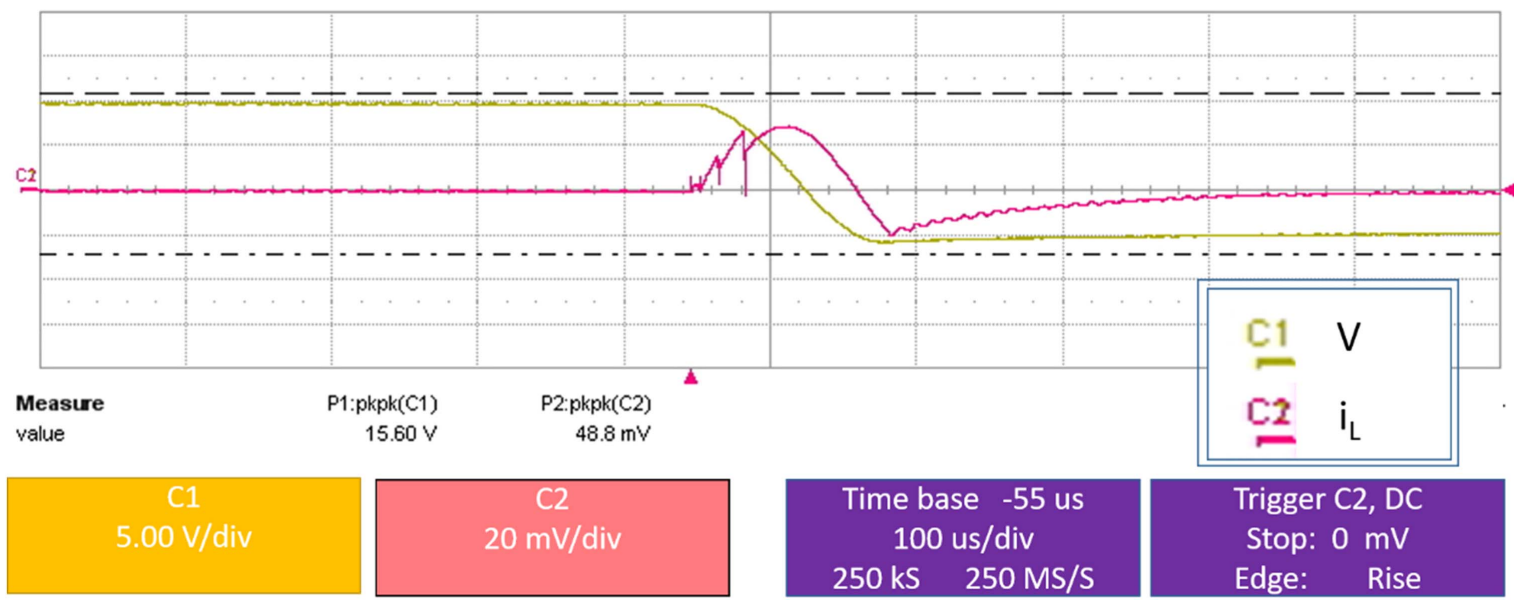

(b)

Figure 13. Experimental result showing (a) LF-P-SSHI waveform using the reed switch as the synchronized switch (b) interfered current flow in respond to the contact bounce during inversion, where $\mathrm{C} 1$ is the voltage waveform and $\mathrm{C} 2$ the current waveform.

\subsection{Non-debounced and debounced experiments}

Firstly, the LF-P-SSHI (figure 6(a)) was taken in to account for its larger scale of current to be observed with CT-2 probes, whilst the inversion factor $\gamma$ is observed to ensure all methods are applicable, meeting the standards.

As a preliminary experiment, two sets of reed-diode pair with opposing polarity was connected LF-P-SSHI, as shown in figure 6(b) to inspect if it is applicable. SSHI waveform appeared as expected as shown in figure 13(a). However, when we look closer in to the inversion period, the current flow shown in figure 13(b) was observed to be interfered by the bouncing contacts, which would hinder the inversion and increase damping with no doubt. The inversion factor $\gamma$ can be obtained as 0.59 , which for the typical value of $0.6-0.8$ mentioned in previous work of Guyomar et al [22], was a bit lame. It could be already predicted that the loaded output using the reed diode would not be optimal. From the resulting waveform of figure 13(b), zooming into the switching instance, three high frequency signals can be observed in the inversion, one attributes to the calling signal of the switch, for the second and third are caused by the re-bound. It was observed that a valley cut showed during the current flow when the second and third closure happens.

Placing in the RC snubber, the current flow was smoothened, as shown in figure 14(a), with only attenuated bouncing interference still appearing, also forming a small valley cut. The inversion ratio was raised and came to 0.69 , which is moderate for the standard of 0.6-0.8. However, the inversion was predicted to be able to be further improved attributing to the loss throughout the resistance.

From the output waveform of figure 14(b), it could be observed that although the peak voltage of the waveform has no obvious change with human observation, but the current spiking was reduced and the valley-cut was held to a smoother curve. It could be also noted that negative current showed after the inversion. Liang et al [23] has a description of the cause. However, the parasitic LC resonance in the 


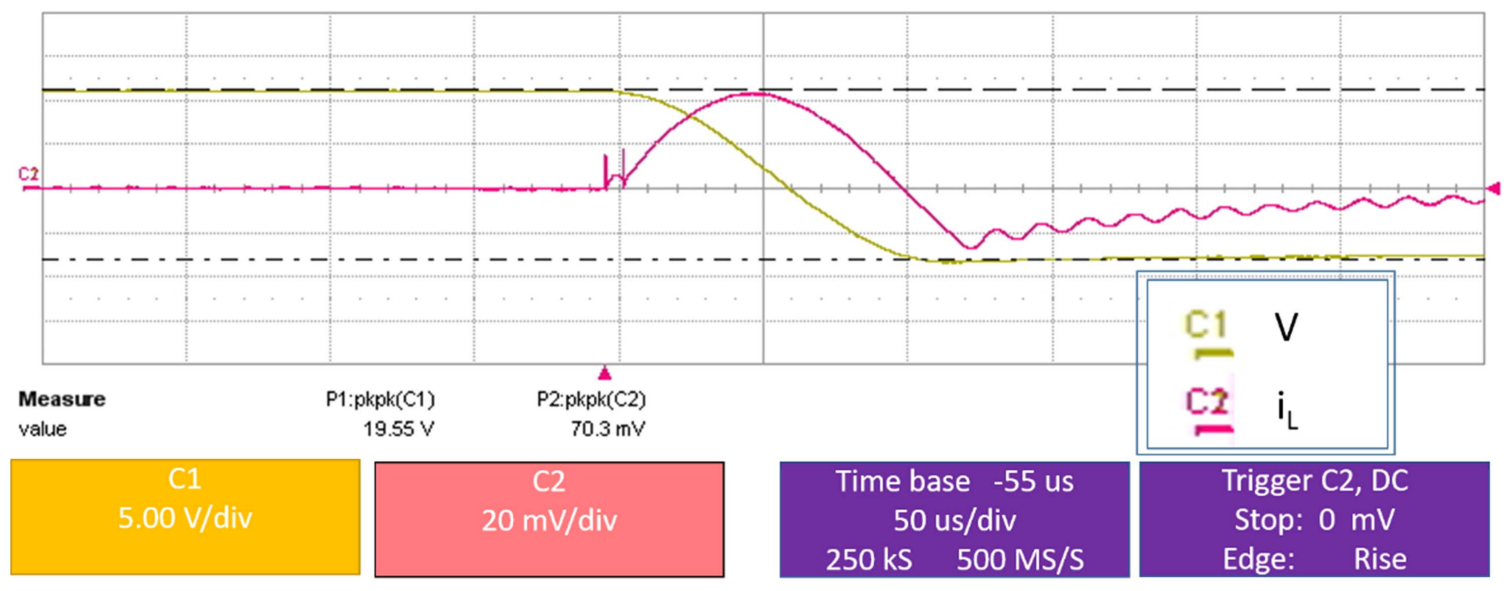

(a)

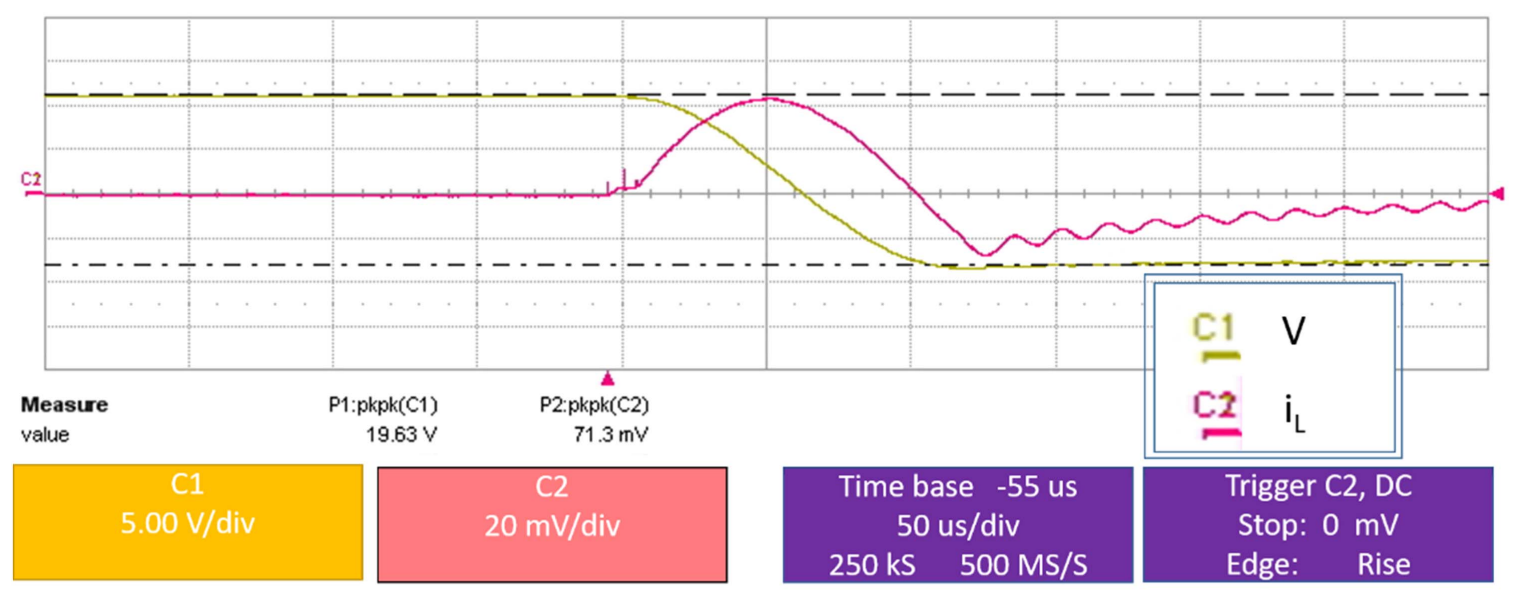

(b)

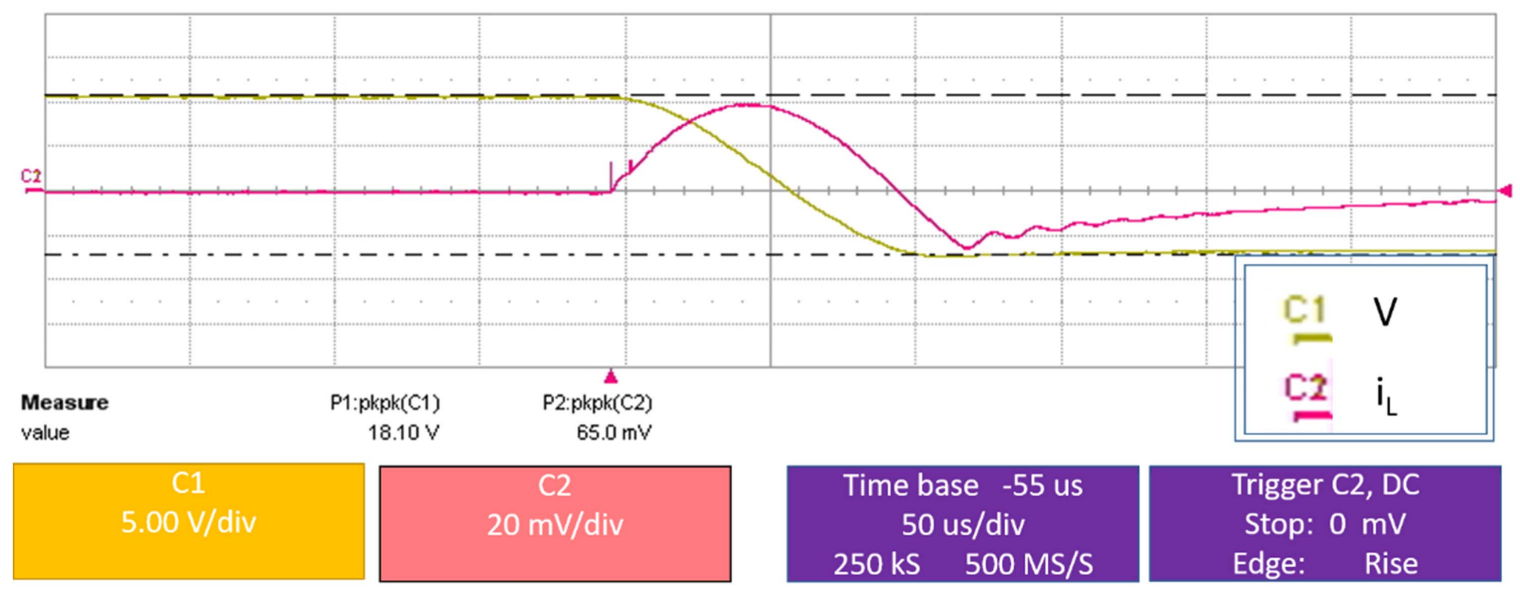

(c)

Figure 14. Voltage $(C 1)$ and current $(C 2)$ waveform of inverting instances current waveform using (a) RDR, (b) RDI, and (c) SCR hybrid switches. 


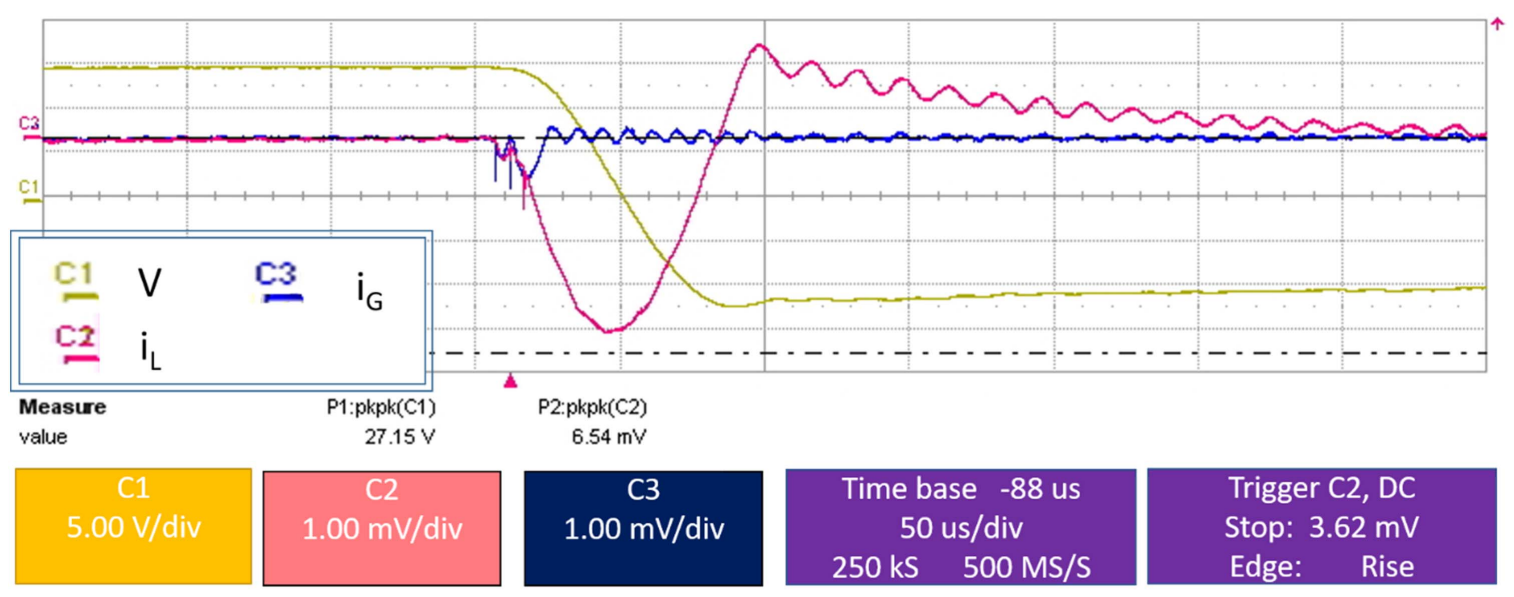

(a)

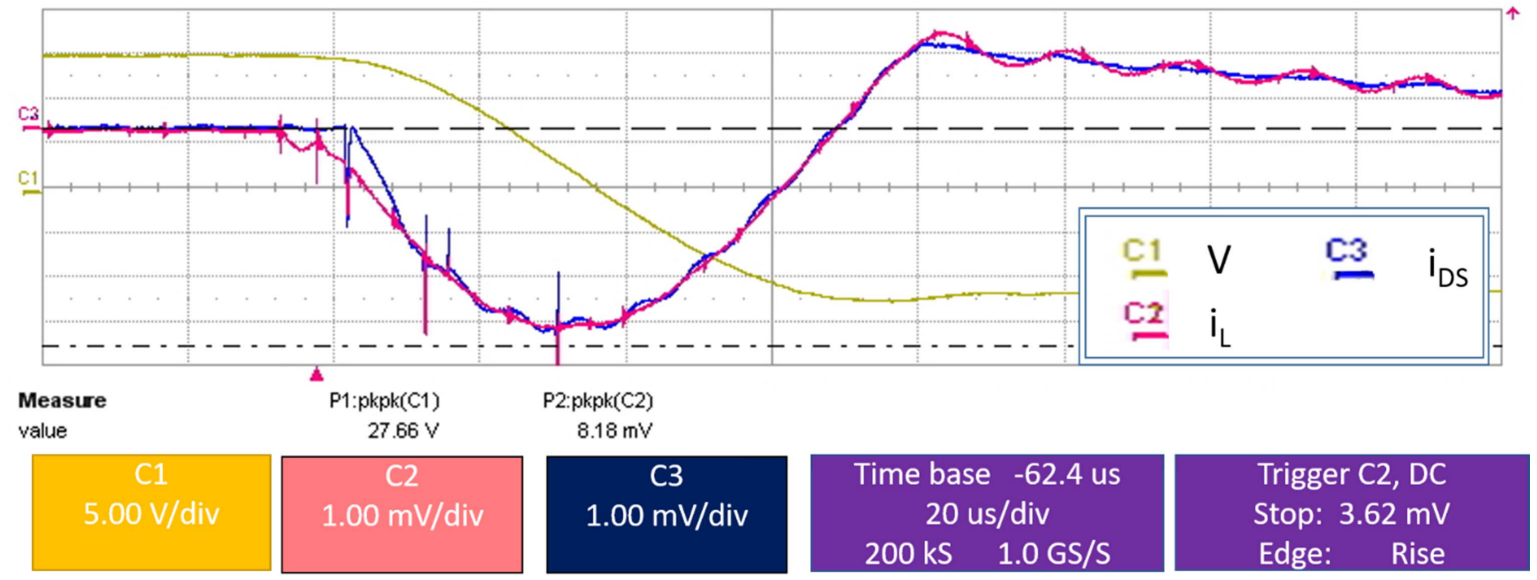

(b)

Figure 15. Voltage and current tracking of LF-P-SSHI inverting instance using RDI hybrid switches where (a) $C 1$ the voltage, $C 2$ the total current, $C 3$ the current into the gate of the MOSFET (b) $C 1$ the voltage, $C 2$ the total current, $C 3$ the current through terminals DS of the MOSFET.

circuit caused the damp down to be prolonged in our experiment.

As we apply SCR in the LF-P-SSHI, with identical exciting circumstances as we applied to the RDC and RDI SSHI, the inversion ratio is 0.66 , as shown in figure $14(\mathrm{c})$, despite slightly lower than that of the RDI, it also showed a smoothened current flow.

\subsection{Experimental confirmations for working mechanisms}

5.3.1. Snubbed switches working mechanism. Again, with a LF-P-SSHI we can observe the current which could be hardly observed when the circuit is loaded. By observing the current, we are able to observe the working mechanism of the snubbed hybrid switch. The current flowing through the gate and the terminals DS were tracked using Tektronix CT-2 probes, so as to understand the working mechanism of the configurations. Figure 15(a) shows the experiment results indicating that the MOSFET, will draw a small portion of the current initially (C3 of figure 15(a)), from the RDI capacitor to start the overall conduction, where $I_{\mathrm{DS}}$ is conducted (figure 15(b)). This we consider the energy dissipation to be caused by the component.

5.3.2. SCR working mechanism. The current flowing into the SCR terminals $\mathrm{G}\left(I_{\mathrm{G}}\right)$ and $\mathrm{A}\left(I_{\mathrm{AK}}\right)$ (figure 11$)$ were tracked using Tektronix CT2 current probes can be found in figure 16, so as to understand the working mechanism of the configurations. As expected, the two routes, $I_{\mathrm{G}}$ and $I_{\mathrm{AK}}$, composed the overall current, while the current flowing into the terminal $G$ was fluctuating due to the chattering. The current was compensated by the route $\mathrm{AK}$, which in summation created a smooth current which we aimed for. This implies that the component loss for the SCR is mostly caused by the inner resistances of the SCR.

\subsection{Power output experiment}

5.4.1. Filter snubbers. Parallel and series SSHI were both applied to determine whether the LC filter could outperform the RC filter in practical applications. In P-SSHI, observing the reed only curve in figure $17(\mathrm{a})$, the loss due to the reed 


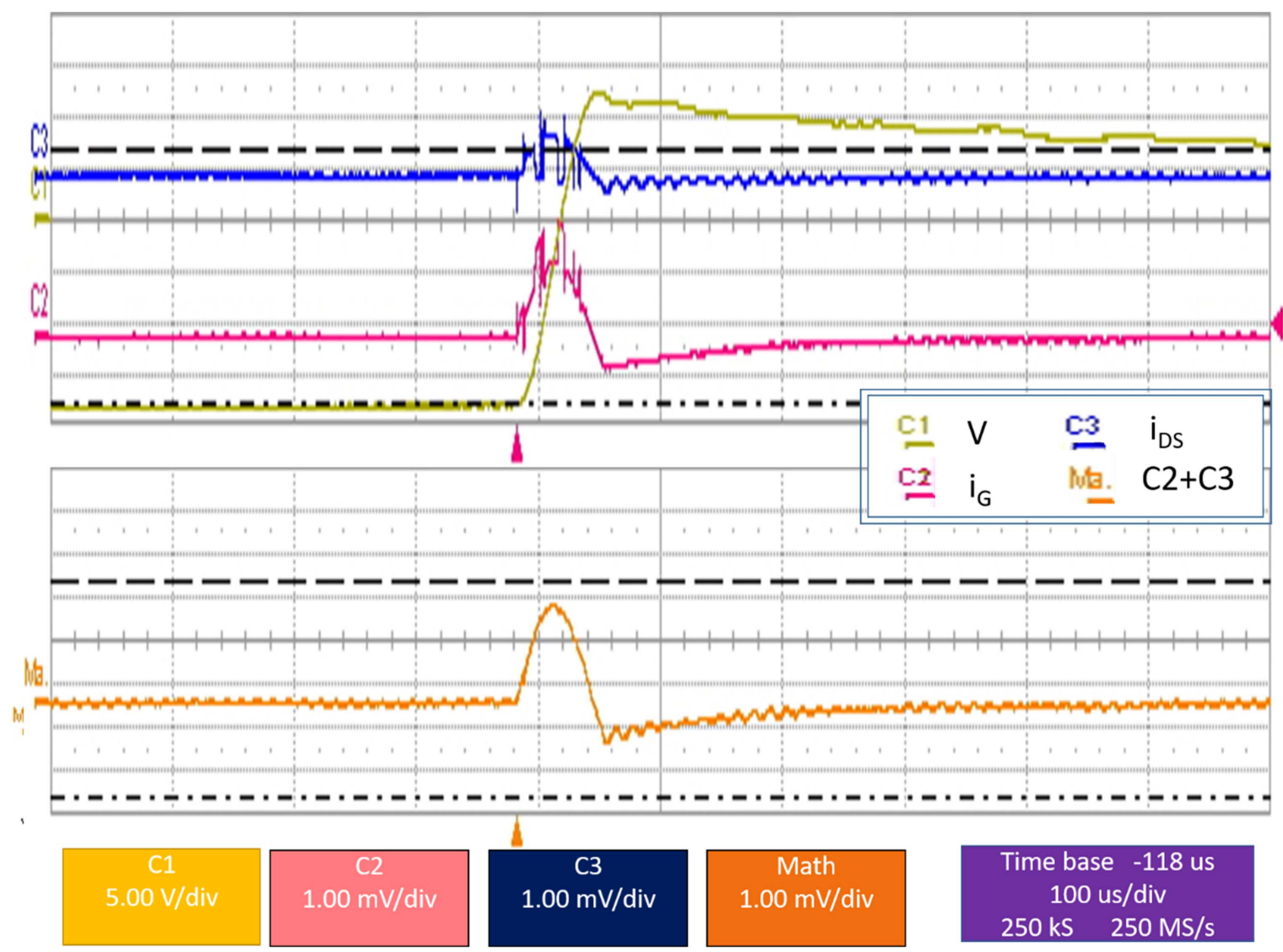

Figure 16. Voltage and current tracking of LF-P-SSHI inverting instance using SCR hybrid switches where $C 1$ is the voltage, $C 2$ the current into the anode of the SCR, $C 3$ the current into the gate terminal of the SCR, Ma the summation of $C 2$ and $C 3$, which sums up to be the overall passing current.

switch is not as dominant as S-SSHI in figure 17(b), which has all the energy flowing through the switches on inversion. In both series and parallel applications, the RDR has a greater power loss, attributing to its voltage division with the loaded impedance. RDI, has a better performance on the other hand. However, we discover that, when loaded with high impedance, the S-SSHI-RDI suffer from poor and unstable switching. It is caused by the divided voltage and limited current that were required to trigger the switches with high impedance load. Figure 18 shows the waveform output of the non-correctly triggered S-SSHI-RDI.

5.4.2. SCR compared to RDI. With beam 2, S-SSHI was used again to test the efficiency of the SCR configuration. To compare with, external powered and also RDI was taken account. From the result in figure 19, we find that the SCR showed to be stable with higher impedances, whilst the RDI show a drop in output right after the optimal impedance. However, it was discovered in figure 20 that when operated in high voltage input and low impedances on series SSHI, SCRs showed unwanted conductions. We can attribute the malfunctioning to the $V_{\mathrm{RG}}$ of the SCR, that is, the maximum reverse voltage than can be applied across gate and cathode terminals, without risk of destructing the PN junction GK. For P0130AA, $V_{\mathrm{RG}}$ is $8 \mathrm{~V}$ minimum, with the reversed gate current of $10 \mu \mathrm{A}$. Despite for the lowered current, $V_{\mathrm{RG}}$ can be increased, SCRs are not feasible with high voltage applications.

\subsection{Phase difference experiment}

An experiment was conducted to investigate the effect of switching phase difference. The theoretical curve equation can be referenced to [21], which the gain $\chi_{\text {para }}$ and $\chi_{\text {series }}$ for the affected output can be further expressed as the equations:

$$
\begin{gathered}
\chi_{\text {para }}=\frac{P_{\text {para,lead }}}{P_{\text {para,opt }}}=\cos ^{2} \theta, \\
\chi_{\text {para }}=\frac{P_{\text {para,lag }}}{P_{\text {para,opt }}}=\frac{[1+\cos \theta-\gamma(1-\cos \theta)]^{2}}{4}
\end{gathered}
$$

and

$$
\chi_{\text {series }}=\frac{P_{\text {series,diff }}}{P_{\text {series,opt }}}=\cos ^{2} \theta .
$$

The relationship between the phase difference and the beam deflection can be found in equation (15). For P-SSHI, the phase leading and lag applies into two different equations (16) and (17). Experimental results fall with the trend agreeing that of the theoretical. In contrast, the S-SSHI was found to fall with a steeper trend, with greater loss. We can attribute the difference to the component damping of 


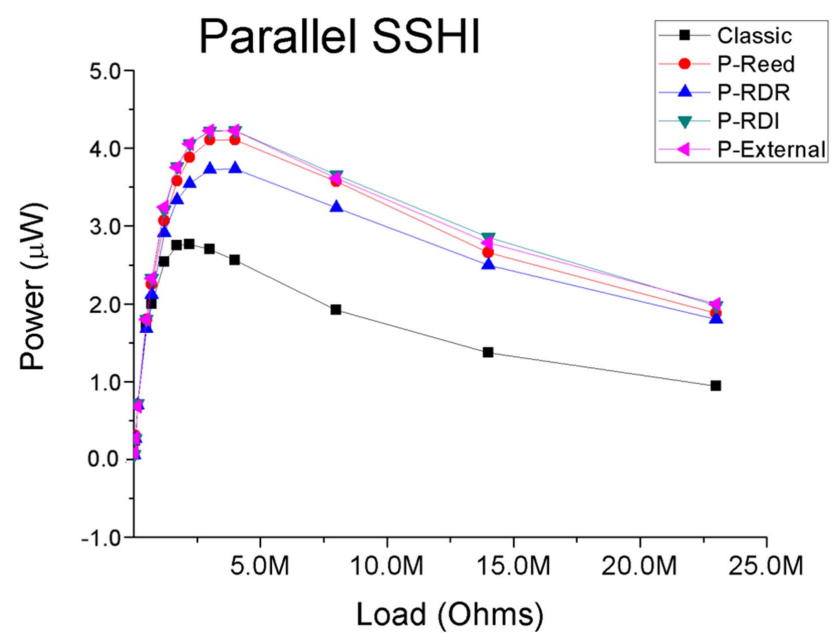

(a)

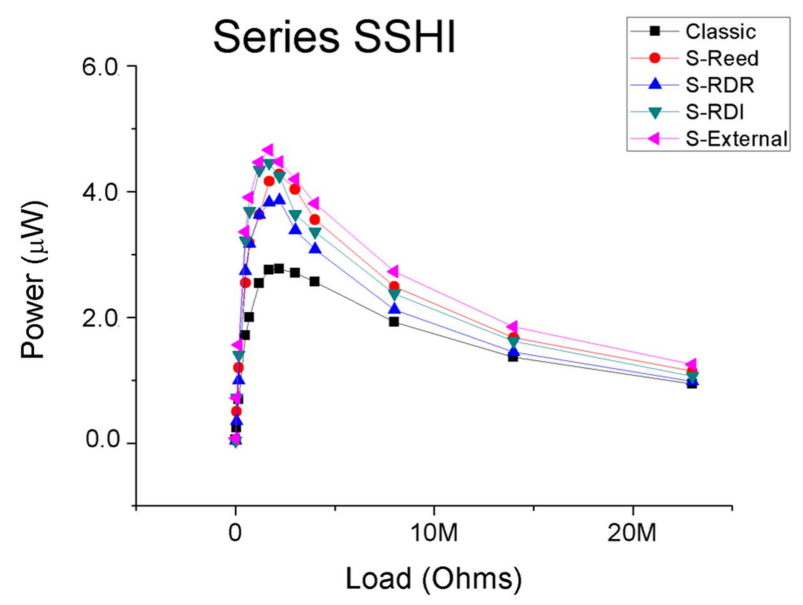

(b)

Figure 17. Power output comparison of SEH (classic), reed diode pair (reed), reed de-bounced with resistance (RDR), reed de-bounced with inductance (RDI) using beam 1 (a) with P-SSHI and (b) S-SSHI. Driven at $0.07 \mathrm{~g}$.

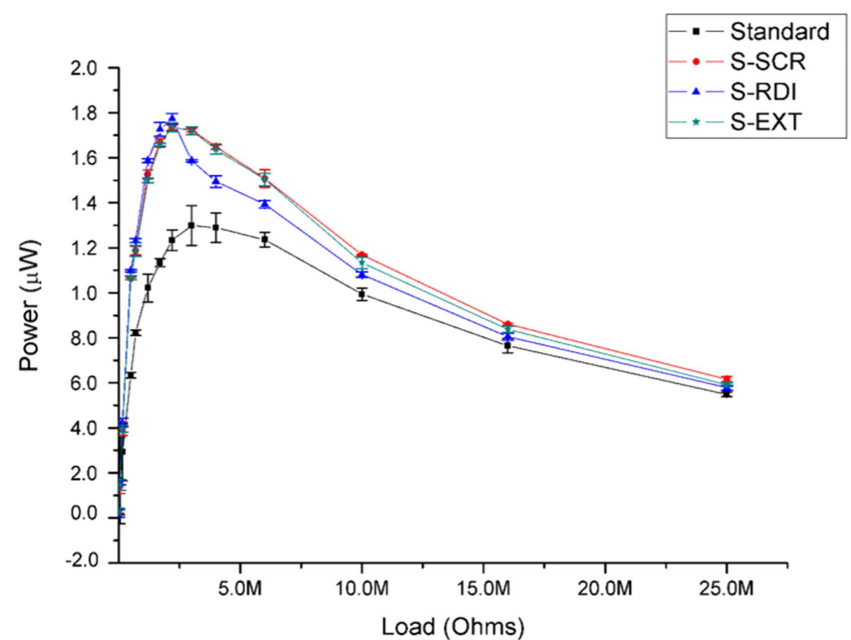

Figure 19. Output comparison of SEH, S-SSHI using SCR, RDI and Externally powered synchronized switches using beam 2 . At the acceleration of $0.03 \mathrm{~g}$.

diodes and MOSFET, which showed to be higher in S-SSHI in experimental results as shown in figure 21 .

\section{Discussion}

To design a synchronized switching system with reed switches, here as follows the description of the design procedure. Before designing, we should consider the operating circumstance-whether it is in a steady or a fluctuating condition. With a constant force, both P-SSHI and S-SSHI can be applied. However, if the input condition varies, P-SSHI is recommended, so that even when there is not enough voltage and displacement, SEH can still be operating. It is also a merit that, when properly designed, P-SSHI can be triggered only when enough voltage is available. It is observed that while supplied voltage below the fully switching theshold, the MOSFET could not work in its linear state, causing more component loss and damping the inversion factor $\gamma$. Thus, SEH has a better efficiency when voltage supply is restricted in a low level. Using a reed switch, one will be able to design a targeting voltage for the switch to be triggered.
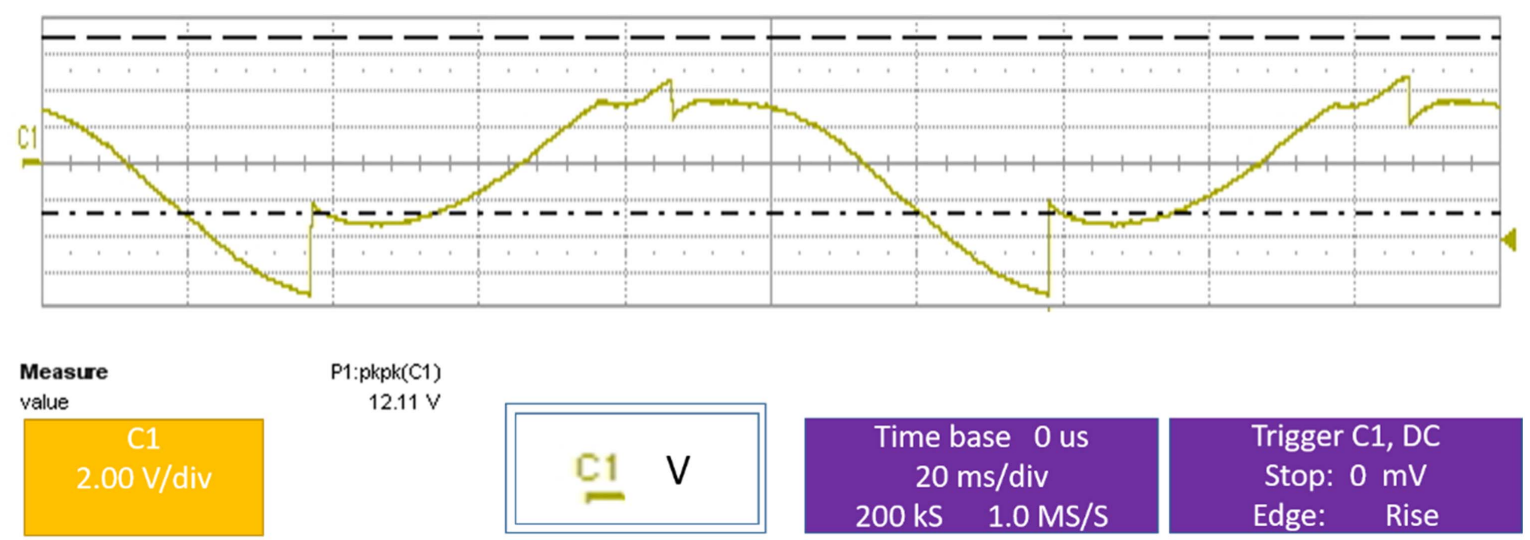

Figure 18. Waveform showing that the electrical switch not correctly triggered when high impedance is loaded in S-SSHI. In the experiment of this work, the value of over-loading impedance is above approximately $3 \mathrm{M} \Omega$. 


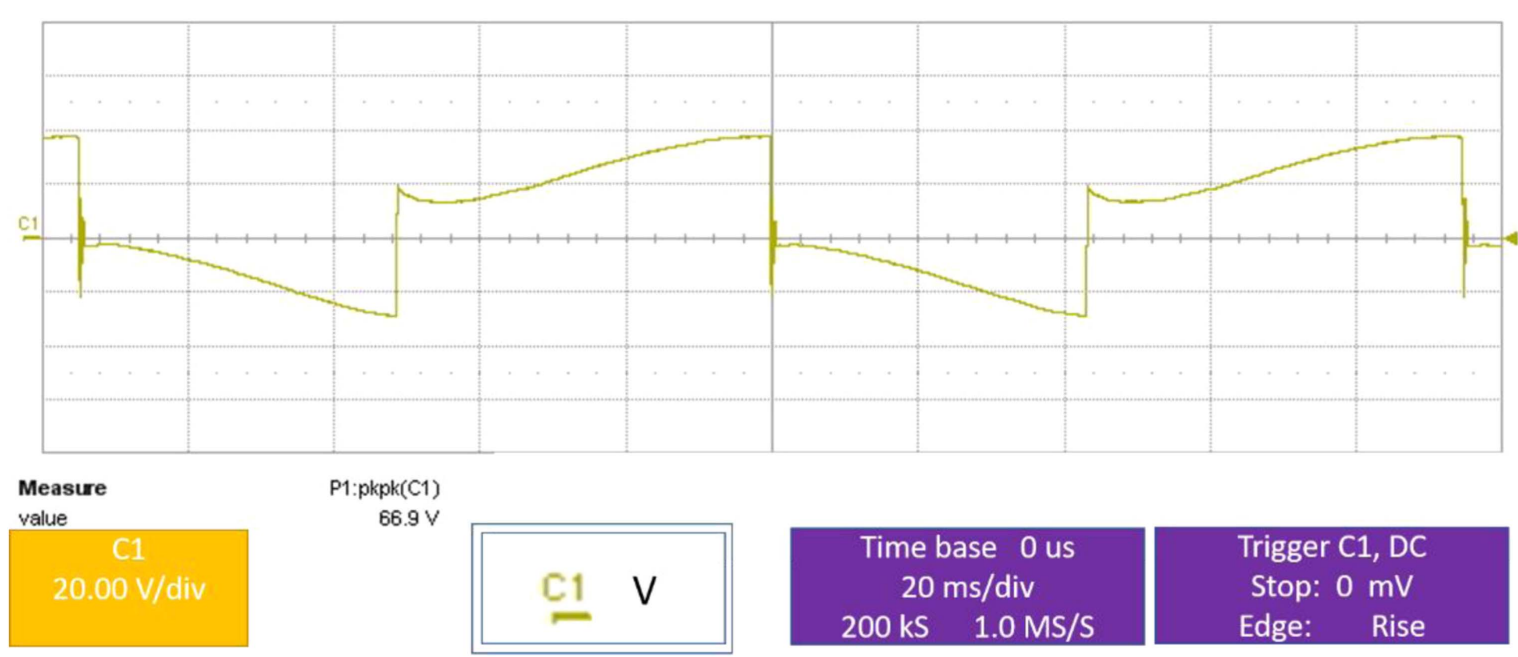

Figure 20. SCR malfunctioning waveform with overdriven voltage.

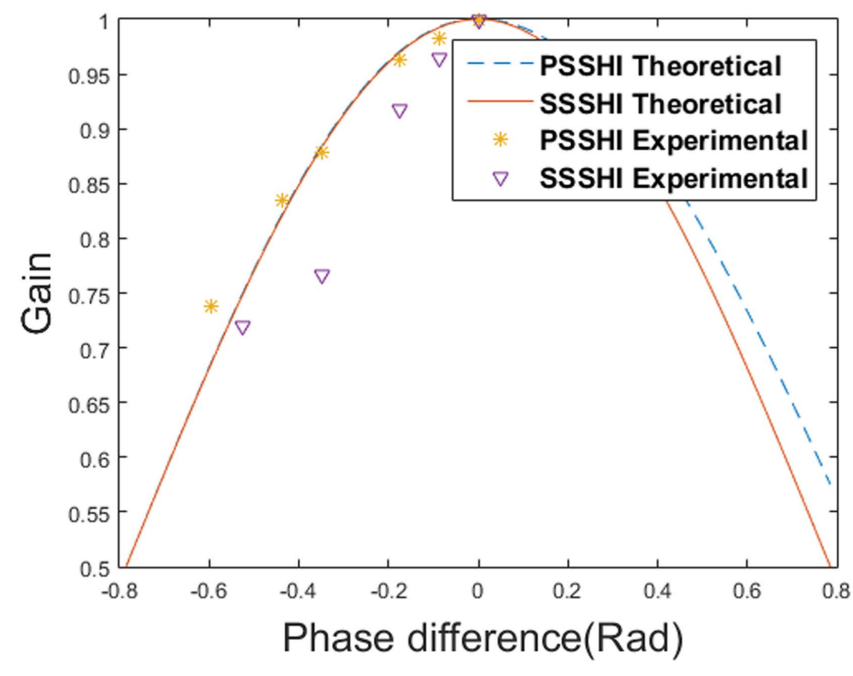

Figure 21. Lag-efficiency experimental results in correspondence to theoretical curve using externally powered switches.

To design the overal system, firstly, the operating frequency should be defined so that the beam dimension can be chosen. To apply reed switches, the cantilever beam substrate should however be able to withstand deformations which should at least exceeed the distance between the PI and DO zone of the reed switch. According to the substrate, dimension and the size of the beam, e.g., micro-sized or bulk sized, the reed switches and magnet pairs could be decided. For microsized cantilever beams such as [24-28], SMD packages are avalable. However, the operating range should be considered, so that the break down voltage and current of the components should not be met. To determine the operating magnet, the closing force in newtons $(\mathrm{N})$ for the reed switch are usually provided in the datasheet, can be used as reference. Some datasheet provide operating ranges in units of Tesla (T). Also it is worth noting that some magnets are designed for reed swtiching as a pair. When the beam substrate and the magnet, serving meanwhile as a proofmass, is chosen, the main beam dimension and thus the acutal operating frequency can then be precisely desgined, considering the operating force and the responding tip deflection. With the knowledge of the tip deflection, we are able to determine favorable switching ranges, including the optimal switching distance and also the available phase difference margin.

The switching distance can then be determined by the magnet-reed switch pair, depending on the shape and size of the PI zone, which is dominated by together the sensitivity of the reed swtich and the magnetic field strength. For convensional glass-capsuled reed switches, overlapping zones which exist between the tip zones and major zones should be averted so as to prevent from false signals. Another point of consideration is the distance between the PI and DO zones. To be conservative, on the switching period should not exceed $50 \%$ of the duty cycle so that both switches would not conduct simultaneously. Which means, when operating in small displacements, the DO zone should not cover all the range of beam tip movement. By referencing [5], we can calculate theo

Table 3. Performance of proposed methods under varying operating circumstances.

\begin{tabular}{|c|c|c|c|}
\hline Operating situations & Reed-diode & SCR-P0130AA & RDI-2N7002T1 \\
\hline Limited voltage/current & (:) & $\odot$ & $:$ \\
\hline Moderate conditions & 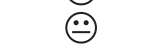 & (:) & (:) \\
\hline High voltage & 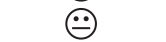 & 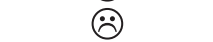 & (:) \\
\hline High current $^{\mathrm{a}}$ & 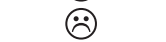 & (:) & (-) \\
\hline
\end{tabular}

${ }^{a}$ Piezoelectric components do not provide high current flow continuously. High current flow is usually caused by the LC resonance on switching, usually on series SSHI with low impedance or SSHI without loads. 
optimal power output with constant driving force, and further derive corresponding tip deflection. We can then derive the reed switched poweroutput with the equations cosidering the time lag effects as below:

$$
\begin{aligned}
& \left\{P_{u, \text { para,reed }}=\frac{R_{L} \omega_{r}^{2} \alpha^{2}\left[u_{M}+d-\gamma\left(u_{M}-d\right)\right]^{2}}{\left[\pi+R_{L} \omega_{R} C_{0}(1-\gamma)\right]^{2}}, u_{M} \geqslant d,\right. \\
& \left\{P_{u, \text { series, reed }}=\frac{4 R_{L} \omega_{r}^{2} \alpha^{2}(1+\gamma)^{2}}{\left[\pi(1-\gamma)+\underset{2}{\left.2 R_{L} \omega_{R} C_{0}(1+\gamma)\right]^{2}}\right.} d^{2}, u_{M} \geqslant d .\right.
\end{aligned}
$$

On different operating conditions, each of the diode has its advantage: the reed-diode configuration, with the simplest components, has better efficiencies on limited power. With limited power, the performances of SCRs and RDI was not ideal. However, when enough power was supplied, both SCR and RDI could work properly. With the ultra-low power SCR, the SCR configuration outperformed RDI in moderate power conditions. When the power is abundant, SCR and RDI both work with great efficiency in P-SSHI. However, for S-SSHI, the electric switch itself endure high voltage or limited current depending on the load. When high cross voltage is met, i.e. low impedance loaded, the SCR meets its breakdown and the mechanism was not feasible. Moreover, when high impedance is loaded, the RDI was not able to collect enough energy to launch, causing unstable switching. It could be observed that a decrease of the output curve showed in response to the impedances in the experimental result. A table 3 was made to give an idea of which configuration to choose under different operating situations.

\section{Conclusion}

In this work, we have proposed a brand new mechanical method to realize the synchronized switching with reed switches. To reduce the contact bounce of the reed switches, three additional methods other than adding simply a diode are proposed: SCRs, RDC, and RDI. Each of the method has its merits when applied in variant conditions: SCRs are available when moderate voltage and high current are supplied, giving an output with stablilty and efficiency. To edure high voltage applications, RDI can be applied, which is an improved version of the RDC. It is, however, limited by the driving energy of the MOSFET, which is determined by the incoming enery flow and the capacitance. When the bouncing of the reed switch is not severe, using only a diode to limit the current flow is also applicable, especially when the drving voltage is not enough, the SCR and RDI methods are not applicable. Since the only threshold voltage is determined by the diode, an appropriate diode would efficiently lower the requirement to operate the synchronized switching technique, i.e., a peak voltage of $0.3 \mathrm{~V}$ is enough to launch the switching. With the debouncing techniques, $100 \%$ efficiency with power output in the scale of aprroximately $20 \mu \mathrm{W}$ can be perfomed in comparison to externally powered parallel SSHI.

\section{Acknowledgments}

We give special thanks to the support from Bourses d'Excellence Eiffel from Campus France, and also the Ministry of Science and Technology of Taiwan (103-2917-I-002-157) for their cross country linking. The authors also gratefully acknowledge the facility support from the Nano-ElectroMechanical-Systems Research Center at National Taiwan University and the funding support from Ministry of Science and Technology, Taiwan though contract number 101-2628E-002-011-MY3 and 104-2923-M-002-010.

\section{References}

[1] Lefeuvre E, Badel A, Richard C and Guyomar D 2004 Highperformance piezoelectric vibration energy reclamation Proc. SPIE 5390379

[2] Guyomar D, Richard C, Lefeuvre E and Petit L 2004 Piezoelectric Non-linear Systems for Standalone Vibration Control and Energy Reclamation (Adaptronic Congress Hildesheim, Germany)

[3] Lefeuvre E, Badel A, Richard C, Petit L and Guyomar D 2006 A comparison between several vibration-powered piezoelectric generators for standalone systems Sensors Actuators A 126 405-16

[4] Richard C, Guyomar D, Audigier D and Ching G 1999 Semipassive damping using continuous switching of a piezoelectric device 1999 Symp. on Smart Structures and Materials pp 104-11

[5] Guyomar D, Sebald G, Pruvost S, Lallart M, Khodayari A and Richard C 2009 Energy harvesting from ambient vibrations and heat J. Intell. Mater. Syst. Struct. 20 609-24

[6] Wu W, Wickenheiser A, Reissman T and Garcia E 2009 Modeling and experimental verification of synchronized discharging techniques for boosting power harvesting from piezoelectric transducers Smart Mater. Struct. 18055012

[7] Lefeuvre E, Badel A, Richard C and Guyomar D 2005 Piezoelectric energy harvesting device optimization by synchronous electric charge extraction J. Intell. Mater. Syst. Struct. 16 865-76

[8] Shen H, Qiu J, Ji H, Zhu K and Balsi M 2010 Enhanced synchronized switch harvesting: a new energy harvesting scheme for efficient energy extraction Smart Mater. Struct. 19115017

[9] Guyomar D and Lallart M 2011 Recent progress in piezoelectric conversion and energy harvesting using nonlinear electronic interfaces and issues in small scale implementation Micromachines 2 274-94

[10] Richard C, Guyomar D and Lefeuvre E 2007 Self-powered electronic breaker with automatic switching by detecting maxima or minima of potential difference between its power electrodes Patent no. FR2005/003000 publication number: WO/2007/063194

[11] Mickaël L and Daniel G 2008 An optimized self-powered switching circuit for non-linear energy harvesting with low voltage output Smart Mater. Struct. 17035030

[12] Lallart M, Lefeuvre É, Richard C and Guyomar D 2008 Selfpowered circuit for broadband, multimodal piezoelectric vibration control Sensors Actuators A 143 377-82

[13] Junrui L and Wei-Hsin L 2009 An improved self-powered switching interface for piezoelectric energy harvesting Int. Conf. on Information and Automation, 2009. ICIA '09 pp 945-50

[14] Yu-Yin C, Vasic D, Costa F, Wen-Jong W and Lee C K 2010 Self-powered piezoelectric energy harvesting device using 
velocity control synchronized switching technique IECON 2010-36th Annual Conf. on IEEE Industrial Electronics Society pp 1785-90

[15] Maiorca F, Giusa F, Trigona C, Andò B, Bulsara A R and Baglio S 2013 Diode-less mechanical H-bridge rectifier for 'zero threshold' vibration energy harvesters Sensors Actuators A 201 246-53

[16] Giusa F, Giuffrida A, Trigona C, Andò B, Bulsara A R and Baglio S 2013 Random mechanical switching harvesting on inductor': a novel approach to collect and store energy from weak random vibrations with zero voltage threshold Sensors Actuators A 198 35-45

[17] Wu Y, Badel A, Formosa F, Liu W and Agbossou A 2014 Nonlinear vibration energy harvesting device integrating mechanical stoppers used as synchronous mechanical switches J. Intell. Mater. Syst. Struct. 25 1658-63

[18] Liu W, Formosa F, Badel A, Wu Y and Agbossou A 2014 Selfpowered nonlinear harvesting circuit with a mechanical switch structure for a bistable generator with stoppers Sensors Actuators A 216 106-15

[19] Liu H, Liang J and Ge C 2015 A mechatronic power boosting design for piezoelectric generators Appl. Phys. Lett. 107 141902

[20] M Electronics 2016 Basic Electrical Parameters of Reed Switch Products available: https://digikey.com/Web\% 20Export/Supplier\%20Content/Meder_374/PDF/ MEDER_Parameters_of_Reed_Switch.pdf

[21] Lallart M, Wu Y-C and Guyomar D 2012 Switching delay effects on nonlinear piezoelectric energy harvesting techniques IEEE Trans. Ind. Electron. 59 464-72

[22] Guyomar D, Sebald D, Pruvost S, Lallart M, Khodayari A and Richard C 2009 Energy harvesting from ambient vibrations and heat J. Intell. Mater. Syst. Struct. 20 609-24

[23] Liang J and Liao W H 2012 Improved design and analysis of self-powered synchronized switch interface circuit for piezoelectric energy harvesting systems IEEE Trans. Ind. Electron. 59 1950-60

[24] Dongna S, Jung-Hyun P, Jyoti A, Song-Yul C, Howard C W III and Dong-Joo K 2008 The design, fabrication and evaluation of a MEMS PZT cantilever with an integrated Si proof mass for vibration energy harvesting J. Micromech. Microeng. 18055017

[25] Liu H, Tay C J, Quan C, Kobayashi T and Lee C 2011 Piezoelectric MEMS energy harvester for low-frequency vibrations with wideband operation range and steadily increased output power J. Microelectromech. Syst. 20 1131-42

[26] Roundy S and Wright P K 2004 A piezoelectric vibration based generator for wireless electronics Smart Mater. Struct. 131131

[27] Lee B S, Lin S C, Wu W J, Wang X Y, Chang P Z and Lee C K 2009 Piezoelectric MEMS generators fabricated with an aerosol deposition PZT thin film J. Micromech. Microeng. 19065014

[28] Shun-Chiu L and Wen-Jong W 2013 Piezoelectric micro energy harvesters based on stainless-steel substrates Smart Mater. Struct. 22045016 